\title{
Anisotropic elastoplastic phase field fracture modeling of 3D printed materials
}

\author{
Pengfei $\mathrm{Li}^{\mathrm{a}}$, Julien Yvonnet ${ }^{\mathrm{a}, *}$, Christelle Combescure ${ }^{\mathrm{a}}$, Hamid Makich ${ }^{\mathrm{b}}$, Mohammed Nouari $^{\mathrm{b}}$ \\ ${ }^{a}$ MSME, Univ Gustave Eiffel, CNRS UMR 8208, F-77454 Marne-la-Vallée, France \\ ${ }^{b}$ Université de Lorraine, CNRS, Arts et Métiers ParisTech, IMT, LEM3 F-88100 Saint Dié des Vosges, France
}

\begin{abstract}
A phase field model for anisotropic, elastoplastic fracture model in layered structures obtained by $3 \mathrm{D}$ printing processes is proposed. An extension of anisotropic phase field to elastoplasticity model is developed. The model is able to describe a transition from quasi-brittle to elastoplastic fracture behaviors depending on the angle of layers in the microstructure with respect to the external loading. Such feature is of special interest to describe the anisotropic fracture behavior in layered 3D printed materials. The present model introduces two phase field variables, one bulk fracture damage and one micro interfacial damage variables, describing two different micro damage mechanisms. Finally, we have proposed an original methodology to identify the macroscopic strain density as a function of the micro interfacial damage variable using numerical homogenization on Representative Volume Elements. Numerical investigations show that the present model is convergent with respect to mesh refinement, and allows to describe complex crack initiation and propagation in layered elastoplastic structures. An experimental comparison is provided to validate the use of such model for 3D printed polymer materials.
\end{abstract}

Keywords: Phase field method, Anisotropic fracture, Elastoplasticity, RVE calculations, 3D printing

\section{Introduction}

The study of fracture in 3D printed materials has gained growing interest in the past decades [1-4], due to its impact in many industrial applications in a wide range of area, such as aerospace [5], biotechnology [6,7], automotive engineering [8] and environmental industry [9].

$3 \mathrm{D}$ printing as an innovative additive manufacturing technology allows to produce 3D polymer components with complex shapes directly from pre-designed digital models without additional tooling or setup. The two major techniques for 3D printing are the Fused Deposition Modelling (FDM) from thermoplastic material [10] and the Selective Laser Sintering (SLS) [11] from polymer or metal powder. For both techniques, the parts are built up layer by layer (see a schematic view of the SLS process in Fig. 1). More specifically in polymer 3D printing processes, porosity can accumulate between each layer of deposited powder or in polymer fused deposition modeling and may induce a preferential crack propagation direction along weak interphase associated with

\footnotetext{
*Corresponding author

Email address: julien.yvonnet@univ-paris-est.fr (Julien Yvonnet)
} 


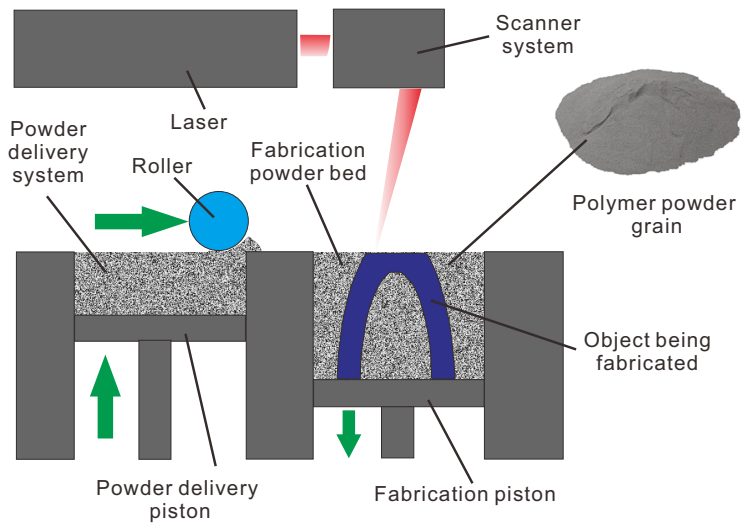

(a)

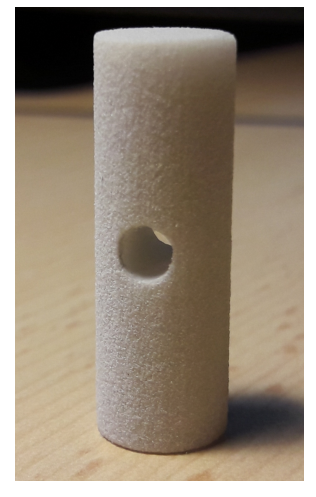

(b)

Figure 1: (a) Schematic view of the 3D printing SLS process (adapted from [13]) and (b) an example of 3D printed part.

the layered structure. Fig. 2 illustrates the effects of orientation of the 3D printed samples on the mechanical properties, from [12]: the behavior can go from brittle when the layers are perpendicular to the loading $\left(0^{\circ}\right)$, to elastoplastic when the layers are parallel to the loading $\left(90^{\circ}\right.$, see Fig. $2(\mathrm{c})$ ). For intermediate orientations, the maximum strain to failure can also be modified.

In the past decades, many models and numerical methods have been proposed to simulate the anisotropic damage failure, including: Finite Elements with anisotropic damage models (e.g. local damage models $[14,15]$ ), anisotropic crack propagation using sharp description of cracks (e.g. FEM remeshing techniques $[16,17]$ and eXtended Finite Element Method (XFEM) [18-20] in anisotropic media), cohesive zone models $[21,22]$ in layered materials. Local damage models are well-known to be associated with mesh-dependency issues and lack of energetic convergence. In methods based on sharp fracture description, crack initiation cannot be easily included, and extension to complex 3D patterns may be highly cumbersome. More specifically, in FEM remeshing techniques, the mesh must be reconstructed after each crack propagation, which might be costly and non-robust for 3D geometries or changes of the crack topology. Cohesive zone modeling is straightforward for crack paths known a priori, but its extension to unknown crack paths may lead to strong mesh dependency and over-estimated cracked areas [23].

Phase field method has emerged as an efficient method for computer simulation of fracture in the past two decades [24-29], and has already been applied and extended in a variety of engineering areas including quasi-brittle fracture [29-32], dynamic fracture [28, 33, 34], fracture in multi physics problems $[35,36]$, hydraulic fracture [37-39], topology optimization for resistance to cracking [4043], interfacial fracture [39, 44-46], bone fracture [47], ductile fracture [48-52] and fracture in micro tomography image-based models of microstructures $[30,53,54]$. In the phase field method, the discrete crack surface is approximated by a diffusive crack representation with an auxiliary scalar damage field. The evolution of the fracture is provided by a variational framework. The phase field modeling offers several advantages, such as the possibility to initiate cracks from undamaged configurations, to handle arbitrary crack networks without specific treatment and use of classical finite elements, a variational framework allowing to include many models or mechanisms, and a mesh-independence due to an appropriate regularization process. Detailed information on the 


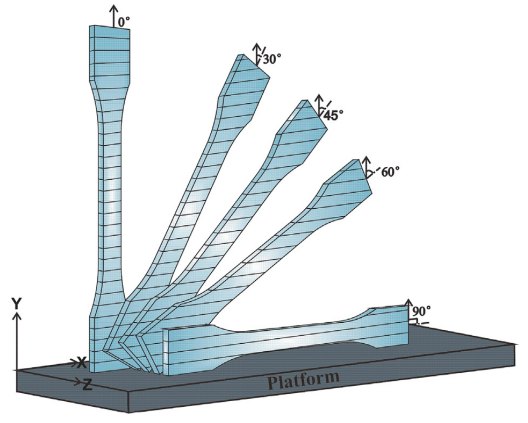

(a)

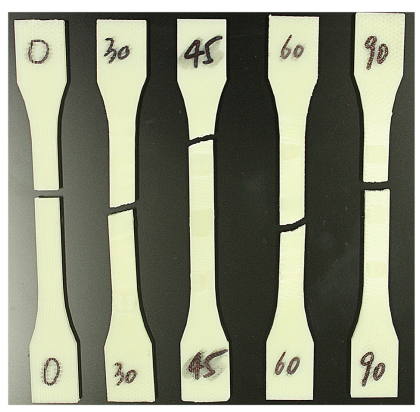

(b)

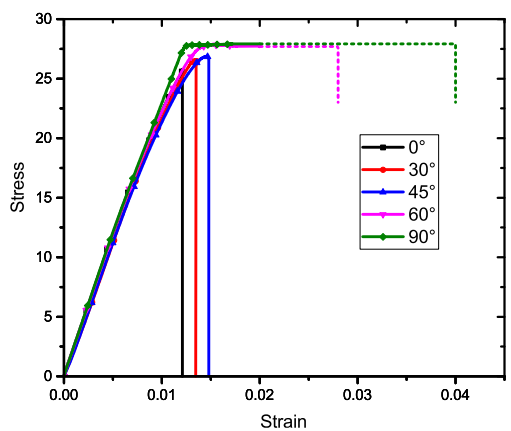

(c)

Figure 2: Experimental tensile test on 3D printed samples [12] exhibiting a transition from quasi-brittle behavior to elastoplastic behavior, depending on the angle between the layers and the tensile direction: (a) samples with different layer orientations, (b) fracture samples and (c) tensile force and displacement curves obtained from uniaxial tensile test.

phase field method can be found e.g. in the review papers $[29,55]$.

Phase field method has been recently extended to anisotropic fracture behaviors, like e.g. in [5661], where an anisotropic fracture energy was employed to simulate crack propagation in anisotropic medias, e.g. in polycrystals [62-65]. In [66, 67], a decomposition of the critical energy release rates was proposed in order to simulated the mixed mode I and II fracture in anisotropic rock-like materials. Alessi and Freddi in [68,69] proposed a phase field approach to simulate failure and complex crack patterns in hybrid laminates, including a competition between fracture of both layers and debonding of the adhesive interface. In [70, 71], a new decomposition for traction-compression decomposition of the strain density energy was proposed to allow applying the phase field method in arbitrary anisotropic elastic media. In [72], Bleyer and Alessi proposed an anisotropic brittle fracture model to simulate the longitudinal/transverse damage in unidirectional fiber-reinforced composites, where a damage-dependent elasticity tensor with respect to two different damage fields was introduced. In [73], an equivalent crack surface energy density function was established to evaluate the fracture state of the homogenized matrix and fiber materials simultaneously.

In the present work, a phase field model has been developed to describe the transition from quasi brittle to elastoplastic behavior in 3D printed materials, with respect to the orientation of the layers. Then, this work introduces several originalities as compared to previous anisotropic phase field models: to our best knowledge, most above mentioned models are restricted to anisotropic quasi brittle models and do not take into account elastoplasticity. The present work then constitutes an important extension in this context. The present model is able to describe a transition from quasibrittle to elastoplastic behavior as a function of the layer orientation in 3D printed layered material. Following [72], we introduce two macroscopic phase field variables, each associated with two distinct damage mechanisms: a bulk layer damage variable, and a variable associated with the damage of the interphase between layers. An original procedure is then introduced to characterize the strain density energy associated with this phase field variable, through computational homogenization on a Representative Volume Element (RVE).

The paper is organized as follows. In Section 2, we develop the anisotropic-elastoplastic phase field model for 3D printed materials. In Section 3, we describe an approach to construct an interfacial damage-dependent elasticity strain density function based on numerical calculations on 

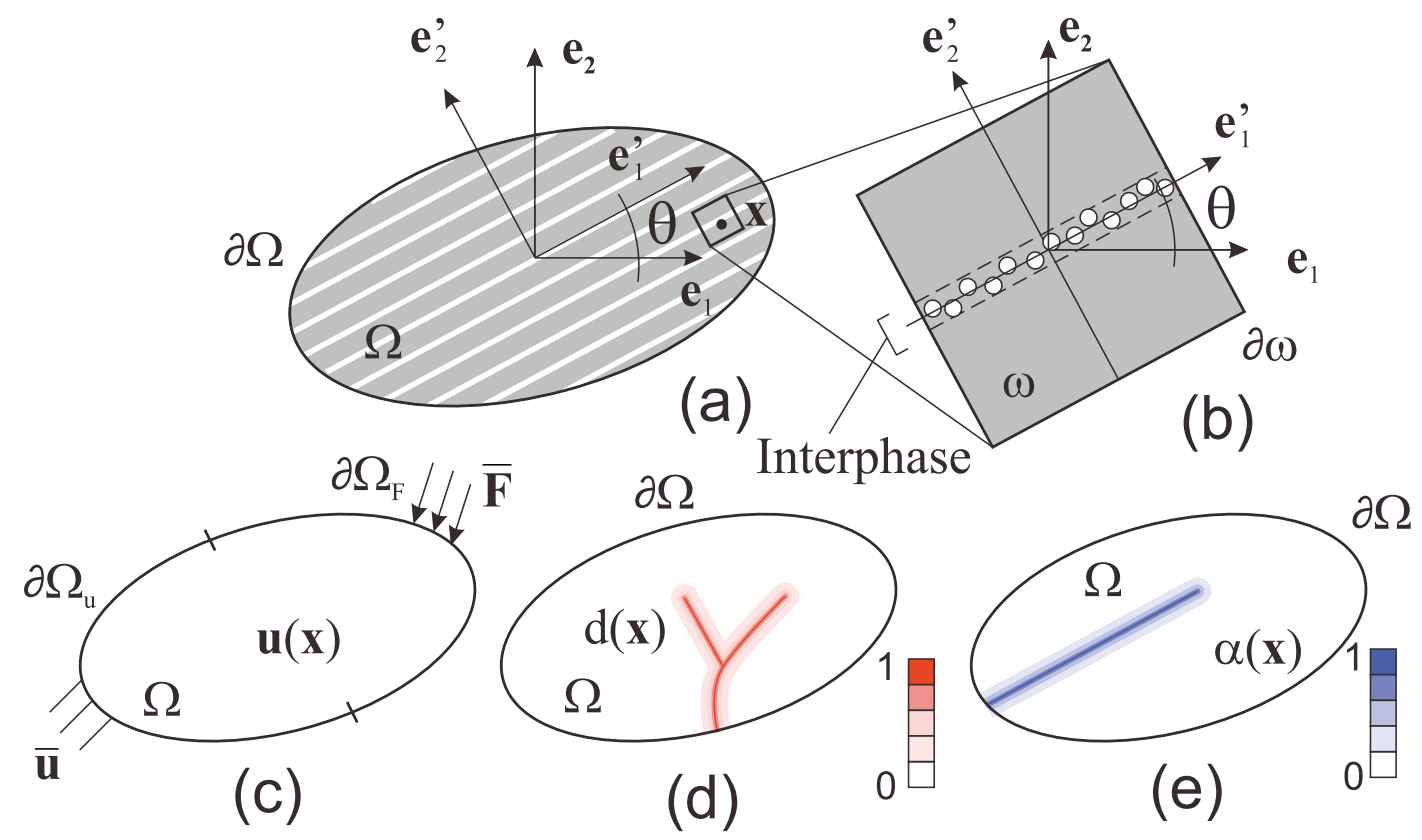

Figure 3: (a) Layered structure; (b) RVE containing one weak interface/interphase in the direction $\mathbf{e}_{1}^{\prime}$; (c) displacement field; (d) bulk layer damage $d(\mathbf{x})$; (e) micro interfacial damage variable $\alpha(\mathbf{x})$.

RVEs. Section 4 provides the FEM discretization and numerical implementation. Finally, numerical examples are presented in Section 5, including numerical investigations and an experimental validation of the model.

\section{Phase field approach to anisotropic elastoplastic damage in $3 \mathrm{D}$ printed materials}

\subsection{Damage variables}

We consider a structure with layered microstructure (see Fig. 3 (a)), as obtained e.g. in 3D printing SLS process. It is assumed that interfaces have weaker mechanical resistance than each layer, then inducing an anisotropic fracture behavior in the direction of the interfaces. In the present work, we define two macroscopic damage variables associated with two distinct fracture mechanisms at the microscale: (a) a classical damage variable $d$ describing the damage due to the cracks in the bulk layers, without a preferential direction and (b) a damage variable $\alpha$ describing the damage of the interfaces at the micro level, associated with an anisotropic damage in the direction of the weak planes.

In Fig. 3 (a) the structure is depicted. The angle between the planes containing the layers and the direction $\mathbf{e}_{1}$ associated with the Cartesian frame is denoted by $\theta$. The structure is associated with an open domain $\Omega \subset \mathbb{R}^{2}$ with boundary $\partial \Omega$, composed of Dirichlet and Neumann parts, denoted by $\partial \Omega_{u}$ and $\partial \Omega_{F}, \partial \Omega=\partial \Omega_{u} \cup \partial \Omega_{F}, \partial \Omega_{u} \cap \partial \Omega_{F}=\emptyset$, where displacements and tractions are prescribed (see Fig. 3 (c)), respectively. The mechanical state of the material within the structure is then defined by the displacement $\mathbf{u}$, bulk fracture $d$ and micro interface fracture $\alpha$ parameters (see Figs. 3 (c), (d) and (e)).

Defining $\mathbf{x} \in \Omega$ as a material point within the structure, a value of $\alpha(\mathbf{x})=1$ indicates that in the neighborhood of $\mathbf{x}$, the interfaces at the micro scale are fully damaged, then inducing an anisotropic 
behavior, while $\alpha(\mathbf{x})=0$ corresponds to an undamaged state of the interfaces. Similarly, $d(\mathbf{x})=1$ and $d(\mathbf{x})=0$ correspond to a fully broken state and undamaged states of the bulk layers. We assume that all layers are parallel. The normal unitary vector to the layers is denoted by $\mathbf{n}^{\alpha}$. We define the projector

$$
\mathbf{P}^{\alpha}=\mathbf{1}-\mathbf{n}^{\alpha} \otimes \mathbf{n}^{\alpha}
$$

such that $\mathbf{v}=\mathbf{P}^{\alpha} \mathbf{u}$ is the projection of the vector $\mathbf{u}$ over $\mathbf{e}_{1}^{\prime}$ (see Fig. 3 (b)) and $\mathbf{1}$ is the second-order identity tensor.

\subsection{Energy density function}

In the following, the fracture is described using a regularized description of the cracks through the smeared damage fields $\alpha$ and $d$ within the phase field fracture method [24-26]. To extend the phase field formulation to anisotropic elastoplastic model, we define the total energy of a cracked structure in the absence of body forces as:

$$
W=\int_{\Omega}\left(\psi^{e}+\psi^{p}+\psi^{d}+\psi^{\alpha}\right) d \Omega-\int_{\partial \Omega_{F}} \overline{\mathbf{F}} \cdot \mathbf{u} d S
$$

where $\psi^{e}$ denotes elastic strain density energy function, $\psi^{p}$ denotes the plastic strain density energy function, $\psi^{d}$ is the bulk layer fracture density function and $\psi^{\alpha}$ is the micro interfacial fracture density function.

We define

$$
\psi^{e}=\frac{1}{2}\left(\varepsilon-\varepsilon^{p}\right): \mathbb{C}(\alpha, d):\left(\varepsilon-\varepsilon^{p}\right)
$$

where $\mathbb{C}(\alpha, d)$ is the damage-dependent elasticity tensor. The elastic strain tensor is defined as:

$$
\varepsilon^{e}=\varepsilon-\varepsilon^{p},
$$

where $\varepsilon=\frac{1}{2}\left(\nabla \mathbf{u}+\nabla^{T} \mathbf{u}\right)$ and $\varepsilon^{p}$ denote respectively the total and the plastic strain tensors. Plastic incompressibility is assumed, i.e. $\operatorname{Tr}\left(\varepsilon^{p}\right)=0, \operatorname{Tr}($.$) being the trace operator.$

We assume that $\mathbb{C}$ depends on $\alpha$ and $d$ in the form:

$$
\mathbb{C}(\alpha, d)=g(d) \overline{\mathbb{C}}^{\alpha}(\alpha),
$$

where we have chosen

$$
g(d)=(1-d)^{2} .
$$

It should be noted that $\overline{\mathbb{C}}^{\alpha}(\alpha)$ is constructed in this work by fully capturing the behavior of the RVE, unlike in [72], where the anisotropic brittle fracture model was based on an empirical damage-dependent elasticity tensor. A detailed description for the construction of $\overline{\mathbb{C}}^{\alpha}(\alpha)$, based on numerical calculations on RVE, is provided in Section 3.

In $(2), \psi^{p}$ is here chosen as

$$
\psi^{p}=\sigma_{y} p+\frac{1}{2} H p^{2},
$$


which describes a linear isotropic hardening law for the plasticity evolution, as shown in Section 2.3, where $\sigma_{y}$ and $H>0$ denote the yield stress and the hardening modulus parameters. In (7), $p$ is an equivalent plastic strain described by the evolution equation

$$
\dot{p}=\sqrt{\frac{2}{3}}\left\|\dot{\boldsymbol{\varepsilon}}^{p}\right\|
$$

where (.) denotes time derivative. In (2), $\psi^{\alpha}$ and $\psi^{d}$ are defined as

$$
\psi^{\alpha}=g_{c}^{\alpha} \gamma^{\alpha}(\alpha), \quad \psi^{d}=g_{c}^{d} \gamma^{d}(d),
$$

where $g_{c}^{\alpha}$ and $g_{c}^{d}$ are the fracture toughnesses with respect to $\alpha$ and $d$, respectively.

The bulk layer crack surface density function for $d$ is defined by:

$$
\gamma^{d}(d)=\frac{d^{2}}{2 \ell_{d}}+\frac{\ell_{d}}{2} \nabla d \cdot \nabla d
$$

where $\ell_{d}$ is a length scale regularization parameter associated to $d$.

To enforce a preferential direction related to the damage induced by the micro interfacial failure, an anisotropic crack surface density function is introduced $[62,63]$ as:

$$
\gamma^{\alpha}(\alpha)=\frac{\alpha^{2}}{2 \ell_{\alpha}}+\frac{\ell_{\alpha}}{2} \omega^{\alpha}:(\nabla \alpha \otimes \nabla \alpha),
$$

where $\ell_{\alpha}$ is the length scale regularization parameter associated to $\alpha$ and

$$
\boldsymbol{\omega}^{\alpha}=\mathbf{1}+\xi^{\alpha} \mathbf{P}^{\alpha}
$$

with $\xi^{\alpha} \gg 1$ being a parameter used to penalize damage along the direction normal to $\mathbf{n}^{\alpha}$.

In the present work, the length scale parameter $\ell_{\alpha}$ and $\ell_{d}$ are simply chosen according to $\ell_{\alpha}=\ell_{d}=2 h_{e}$, where $h_{e}$ is the minimal element size. However, it is worth noting that these parameters can be interpreted as material parameters (see a discussion in [74]). Possible improvements may imply the use of recent length-free formulations as e.g. proposed in [75] or in [76], where a length-scale insensitive anisotropic Phase Field method considering distinct softening behaviors for isotropic matrix and anisotropic fibers has been proposed.

\subsection{Variational formulation}

In this section, we employ the variational framework for fracture as introduced in $[46,51,77]$ to derive the evolution criteria of the proposed model. The variational framework involves: irreversibility condition, stability condition and energy balance. In this framework, stability condition provides mechanical balance equation, damage and plastic criteria. The energy balance provides damage consistencies and plastic flow rule.

\subsubsection{Irreversibility condition}

The irreversibility condition for both damage variables is expressed as

$$
\begin{aligned}
& \dot{d} \geq 0, \quad 0 \leq d \leq 1 \\
& \dot{\alpha} \geq 0, \quad 0 \leq \alpha \leq 1
\end{aligned}
$$


In the present work, (13) is prescribed using a history function [26] (see section 2.4), while (14) is prescribed numerically by:

$$
\alpha^{n+1}(\mathbf{x})=\left\{\begin{array}{l}
\alpha_{\text {trial }}^{n+1}(\mathbf{x}) \text { if } \alpha_{\text {trial }}^{n+1}(\mathbf{x}) \geq \alpha^{n}(\mathbf{x}) \\
\alpha^{n}(\mathbf{x}) \text { if } \alpha_{\text {trial }}^{n+1}(\mathbf{x})<\alpha^{n}(\mathbf{x})
\end{array}\right.
$$

where $\alpha_{\text {trial }}^{n+1}(\mathbf{x})$ is the value of $\alpha$ obtained at one point $\mathbf{x}$ at time step $n+1$ after solving the micro interfacial damage problem (44) and $\alpha^{n}(\mathbf{x})$ is the value of $\alpha$ obtained at one point $\mathbf{x}$ at time step $n$, and $\alpha^{n+1}(\mathbf{x})$ is the corrected value.

\subsubsection{First-order stability condition}

We first define the directional derivative as:

$$
D_{\mathbf{v}} f(\mathbf{u})=\left[\frac{d}{d h} f(\mathbf{u}+h \mathbf{v})\right]_{h=0} .
$$

The first order stability condition (see [78-80]) is expressed by:

$$
D_{\delta \mathbf{u}} W+D_{\delta p} W+D_{\delta d} W+D_{\delta \alpha} W \geq 0 .
$$

Using (2), (17) yields:

$$
\begin{aligned}
& \int_{\Omega} \boldsymbol{\sigma}: \boldsymbol{\varepsilon}^{e}(\delta \mathbf{u}) d \Omega+\int_{\Omega}\left(-\sqrt{\frac{3}{2}} \boldsymbol{\sigma}: \hat{\mathbf{n}}+\frac{\partial \psi^{p}}{\partial p}\right) \delta p d \Omega+\int_{\Omega}\left(D_{\delta d} \psi^{e}+D_{\delta d} \psi^{d}\right) d \Omega \\
& +\int_{\Omega}\left(D_{\delta \alpha} \psi^{e}+D_{\delta \alpha} \psi^{\alpha}\right) d \Omega-\int_{\partial \Omega_{F}} \overline{\mathbf{F}} \cdot \delta \mathbf{u} d S \geq 0
\end{aligned}
$$

where the Cauchy stress is given by

$$
\boldsymbol{\sigma}=\frac{\partial \psi^{e}}{\partial \varepsilon^{e}}=g(d) \overline{\mathbb{C}}^{\alpha}(\alpha): \varepsilon^{e}
$$

and $\hat{\mathbf{n}}$ is a unit tensor in the direction of the plastic flow. Then, the following results stem out:

- For $\delta p=\delta d=\delta \alpha=0$, we obtain:

$$
\int_{\Omega} \boldsymbol{\sigma}: \boldsymbol{\varepsilon}^{e}(\delta \mathbf{u}) d \Omega-\int_{\partial \Omega_{F}} \overline{\mathbf{F}} \cdot \delta \mathbf{u} d S=0
$$

which is the weak form of the equilibrium equation.

- For $\delta d=\delta \alpha=0$ and $\delta \mathbf{u}=\mathbf{0}$, we obtain

$$
\int_{\Omega}\left(-\sqrt{\frac{3}{2}} \boldsymbol{\sigma}: \hat{\mathbf{n}}+\frac{\partial \psi^{p}}{\partial p}\right) \delta p d \Omega \geq 0 .
$$

For $J_{2}$-plasticity, this expression leads to

$$
\int_{\Omega}\left(\sqrt{\frac{3}{2}}\left\|\boldsymbol{\sigma}_{\mathrm{dev}}\right\|-\frac{\partial \psi^{p}}{\partial p}\right) \delta p d \Omega \leq 0
$$


corresponding to the weak form of the plasticity yield criterion; $\boldsymbol{\sigma}_{\mathrm{dev}}:=\boldsymbol{\sigma}-\frac{1}{3} \operatorname{Tr}(\boldsymbol{\sigma}) \mathbf{1}$ is the deviatoric stress tensor. The associated Euler-Lagrange equation is expressed as:

$$
\mathcal{F}^{p}=\sqrt{\frac{3}{2}}\left\|\boldsymbol{\sigma}_{\operatorname{dev}}\right\|-\frac{\partial \psi^{p}}{\partial p} \leq 0 \text { in } \Omega
$$

which is the classical von Mises yield criterion.

- For $\delta p=\delta \alpha=0$ and $\delta \mathbf{u}=\mathbf{0}$ :

$$
\int_{\Omega}\left(D_{\delta d} \psi^{e}+D_{\delta d} \psi^{d}\right) d \Omega \geq 0
$$

which is the weak form of the bulk damage criterion.

- For $\delta p=\delta d=0$ and $\delta \mathbf{u}=\mathbf{0}$, we obtain:

$$
\int_{\Omega}\left(D_{\delta \alpha} \psi^{e}+D_{\delta \alpha} \psi^{\alpha}\right) d \Omega \geq 0
$$

defining the weak form of the interfacial damage criterion.

\subsubsection{Energy balance}

Following a procedure analogous to the treatment of the stability condition, the energy balance condition leads to

$$
\begin{aligned}
& -\int_{\Omega} \boldsymbol{\sigma}: \varepsilon^{e}(\dot{\mathbf{u}}) d \Omega+\int_{\Omega}\left(\sqrt{\frac{3}{2}} \boldsymbol{\sigma}: \hat{\mathbf{n}}-\frac{\partial \psi^{p}}{\partial p}\right) \dot{p} d \Omega-\int_{\Omega}\left(D_{\dot{d}} \psi^{e}+D_{\dot{d}} \psi^{d}\right) d \Omega \\
& -\int_{\Omega}\left(D_{\dot{\alpha}} \psi^{e}+D_{\dot{\alpha}} \psi^{\alpha}\right) d \Omega+\int_{\partial \Omega_{F}} \overline{\mathbf{F}} \cdot \dot{\mathbf{u}} d S=0 .
\end{aligned}
$$

The following cases are analyzed:

- For $\dot{\mathbf{u}}=\mathbf{0}, \dot{d}=0$ and $\dot{\alpha}=0$, the plasticity consistency condition is obtained:

$$
\mathcal{F}^{p} \dot{p}=0 \text {. }
$$

- For $\dot{\mathbf{u}}=\mathbf{0}, \dot{p}=0$ and $\dot{\alpha}=0$, the bulk damage consistency condition can be written in the form:

$$
\mathcal{F}^{d} \dot{d}=0
$$

where $\mathcal{F}^{d}$ will be specified in section 2.4 .

- For $\dot{\mathbf{u}}=\mathbf{0}, \dot{p}=0$ and $\dot{d}=0$, the interfacial damage consistency condition can be written in the form:

$$
\mathcal{F}^{\alpha} \dot{\alpha}=0
$$

where $\mathcal{F}^{\alpha}$ will be specified in section 2.4 . 


\subsubsection{Alternate minimization}

A staggered alternate minimization algorithm is applied in this section. With the total energy (2) at hand, the alternate minimization follows.

- Minimization with respect to the displacement field:

$$
D_{\delta \mathbf{u}} W=0
$$

leads to

$$
\int_{\Omega} \boldsymbol{\sigma}: \boldsymbol{\varepsilon}^{e}(\delta \mathbf{u}) d \Omega-\int_{\partial \Omega_{F}} \overline{\mathbf{F}} \cdot \delta \mathbf{u} d S=0
$$

which corresponds to the weak form of the mechanical problem to be solved for $\mathbf{u}$, given $d$ and $\alpha$.

- Minimization with respect to the equivalent plastic strain:

$$
D_{\delta p} W=\int_{\Omega}\left(-\sqrt{\frac{3}{2}} \boldsymbol{\sigma}: \hat{\mathbf{n}}+\frac{\partial \psi^{p}}{\partial p}\right) \delta p d \Omega=0
$$

which is the weak form of the plastic yield criterion (21) which has to be satisfied for $\dot{p} \geq 0$. In the present work, this condition is handled by a return-mapping algorithm (see [81]). In [77] a regularization term was introduced in the total energy and the above equation was verified through solving a global problem for $p$. Here we do not adopt this approach and treat this criterion as a local one (at Gauss integration points). (31) and (32) are solved together using the return-mapping algorithm [81].

- Minimization with respect to the bulk damage field:

$$
D_{\delta d} W=\int_{\Omega}\left(D_{\delta d} \psi^{e}+D_{\delta d} \psi^{d}\right) d \Omega=0,
$$

which corresponds to the global problem to be solved to find the field $d(\mathbf{x})$, given $\mathbf{u}, p$ and $\alpha$.

- Minimization with respect to the interfacial damage field:

$$
D_{\delta \alpha} W=\int_{\Omega}\left(D_{\delta \alpha} \psi^{e}+D_{\delta \alpha} \psi^{\alpha}\right) d \Omega=0,
$$

which corresponds to the global problem to be solved to find the field $\alpha(\mathbf{x})$, given $\mathbf{u}, p$ and $d$.

\subsection{Governing equations}

The associated Euler-Lagrange equations to (31) are given by:

$$
\left\{\begin{array}{c}
\nabla \cdot \boldsymbol{\sigma}=\mathbf{0} \text { in } \Omega, \\
\mathbf{u}=\overline{\mathbf{u}} \text { on } \partial \Omega_{u}, \\
\boldsymbol{\sigma} \mathbf{n}=\overline{\mathbf{F}} \text { on } \partial \Omega_{F} .
\end{array}\right.
$$


We can re-write (23) and (8) to obtain the plasticity evolution equations:

$$
\begin{aligned}
& \mathcal{F}^{p}=\sqrt{\frac{3}{2}}\left\|\boldsymbol{\sigma}_{\mathrm{dev}}\right\|-\left(\sigma_{y}+H p\right) \leq 0, \\
& \dot{\boldsymbol{\varepsilon}}^{p}=\dot{p} \sqrt{\frac{3}{2}} \frac{\boldsymbol{\sigma}_{\mathrm{dev}}}{\left\|\boldsymbol{\sigma}_{\mathrm{dev}}\right\|} \text { with } \dot{p} \geq 0 .
\end{aligned}
$$

Using (33) and the property:

$$
(\Delta d) \delta d=\nabla \cdot(\nabla d \delta d)-\nabla d \cdot \nabla(\delta d)
$$

as well as the divergence theorem and $\nabla d \cdot \mathbf{n}=0$, we obtain the weak form of the bulk damage problem as:

$$
\int_{\Omega}\left(\left\{-2(1-d) \mathcal{H}+\frac{g_{c}^{d} d}{\ell_{d}}\right\} \delta d+g_{c}^{d} \ell_{d} \nabla d \cdot \nabla(\delta d)\right) d \Omega=0,
$$

where

$$
\mathcal{H}=\max _{s \in[0, t]} \psi_{0}^{e}(\mathbf{u}, s) \quad \text { with } \quad \psi_{0}^{e}=\frac{1}{2} \varepsilon^{e}: \overline{\mathbb{C}}^{\alpha}(\alpha): \varepsilon^{e}
$$

is a history function to prescribe the irreversibility of bulk damage field. Then, the corresponding Euler-Lagrange equations to (39) are given by:

$$
\left\{\begin{array}{l}
\frac{g_{c}^{d}}{\ell_{d}}\left(d-\ell_{d}^{2} \Delta d\right)=2(1-d) \mathcal{H} \\
\nabla d \cdot \mathbf{n}=0 \text { on } \partial \Omega \\
d=1 \text { on } \Gamma^{d}
\end{array}\right.
$$

From (28) we identify

$$
\mathcal{F}^{d}=\frac{g_{c}^{d}}{\ell_{d}}\left(d-\ell_{d}^{2} \Delta d\right)-2(1-d) \mathcal{H} .
$$

Similarly, we can obtain the weak form of the interfacial damage problem as:

$$
\int_{\Omega}\left[\frac{1}{2} g(d) \varepsilon^{e}: \frac{\partial \overline{\mathbb{C}}^{\alpha}}{\partial \alpha}: \varepsilon^{e} \delta \alpha+\frac{g_{c}^{\alpha}}{\ell_{\alpha}} \alpha \delta \alpha+g_{c}^{\alpha} \ell_{\alpha} \boldsymbol{\omega}^{\alpha}:(\nabla \alpha \otimes \nabla \delta \alpha)\right] d \Omega=0,
$$

the corresponding Euler-Lagrange equations to (43) are given by:

$$
\left\{\begin{array}{l}
\frac{1}{2} g(d) \boldsymbol{\varepsilon}^{e}: \frac{\partial \overline{\mathbb{C}}^{\alpha}}{\partial \alpha}: \boldsymbol{\varepsilon}^{e}+\frac{g_{c}^{\alpha}}{\ell_{\alpha}} \alpha-g_{c}^{\alpha} \ell_{\alpha} \boldsymbol{\omega}^{\alpha}: \nabla \nabla \boldsymbol{\alpha}=0 \\
\nabla \alpha \cdot \mathbf{n}=0 \text { on } \partial \Omega, \\
\alpha=1 \text { on } \Gamma^{\alpha},
\end{array}\right.
$$


Table 1: Governing equations of the anisotropic elastoplastic phase field model.

\begin{tabular}{ll}
\hline Irreversibility & $\dot{d} \geq 0,0 \leq d \leq 1, \quad \dot{\alpha} \geq 0,0 \leq \alpha \leq 1$ \\
Mechanical balance & $\nabla \cdot \boldsymbol{\sigma}=\mathbf{0}$ in $\Omega$ \\
& $\mathbf{u}=\overline{\mathbf{u}}$ on $\partial \Omega_{u}, \boldsymbol{\sigma} \mathbf{n}=\overline{\mathbf{F}}$ on $\partial \Omega_{F}$ \\
Constitutive law & $\boldsymbol{\sigma}=g(d) \overline{\mathbb{C}}^{\alpha}(\alpha): \boldsymbol{\varepsilon}^{e}$ \\
Bulk damage criterion & $\frac{g_{c}^{d}}{\ell_{d}}\left(d-\ell_{d}^{2} \Delta d\right)-2(1-d) \mathcal{H} \geq 0$ \\
& $\mathcal{H}=\max _{s \in[0, t]} \psi_{0}^{e}(\mathbf{u}, s)$ with $\psi_{0}^{e}=\frac{1}{2} \varepsilon^{e}: \overline{\mathbb{C}}^{\alpha}(\alpha): \boldsymbol{\varepsilon}^{e}$ \\
Bulk damage consistency & $\left(\frac{g_{c}^{d}}{\ell_{d}}\left(d-\ell_{d}^{2} \Delta d\right)-2(1-d) \mathcal{H}\right) \dot{d}=0$ \\
Interfacial damage criterion & $\frac{1}{2} g(d) \boldsymbol{\varepsilon}^{e}: \frac{\partial \overline{\mathbb{C}}^{\alpha}}{\partial \alpha}: \boldsymbol{\varepsilon}^{e}+\frac{g_{c}^{\alpha}}{\ell_{\alpha}} \alpha-g_{c}^{\alpha} \ell_{\alpha} \boldsymbol{\omega}^{\alpha}: \nabla \nabla \boldsymbol{\alpha} \geq 0$ \\
Interfacial damage consistency & $\left(\frac{1}{2} g(d) \boldsymbol{\varepsilon}^{e}: \frac{\partial \overline{\mathbb{C}}^{\alpha}}{\partial \alpha}: \boldsymbol{\varepsilon}^{e}+\frac{g_{c}^{\alpha}}{\ell_{\alpha}} \alpha-g_{c}^{\alpha} \ell_{\alpha} \boldsymbol{\omega}^{\alpha}: \nabla \nabla \boldsymbol{\alpha}\right) \dot{\alpha}=0$ \\
Plastic yield criterion & $\mathcal{F}^{p}=\sqrt{\frac{3}{2}}\left\|\boldsymbol{\sigma}_{\mathrm{dev}}\right\|-\left(\sigma_{y}+H p\right) \leq 0$, \\
Plastic flow rule & $\dot{\boldsymbol{\varepsilon}}^{p}=\dot{p} \sqrt{\frac{3}{2}} \frac{\boldsymbol{\sigma}_{\mathrm{dev}}}{\left\|\boldsymbol{\sigma}_{\mathrm{dev}}\right\|}$ with $\dot{p} \geq 0$ \\
\hline
\end{tabular}

where $(\nabla \nabla \boldsymbol{\alpha})_{i j}=\frac{\partial^{2} \alpha}{\partial x_{i} \partial x_{j}}$. From (29) we identify

$$
\mathcal{F}^{\alpha}=\frac{1}{2} g(d) \varepsilon^{e}: \frac{\partial \overline{\mathbb{C}}^{\alpha}}{\partial \alpha}: \varepsilon^{e}+\frac{g_{c}^{\alpha}}{\ell_{\alpha}} \alpha-g_{c}^{\alpha} \ell_{\alpha} \boldsymbol{\omega}^{\alpha}: \nabla \nabla \boldsymbol{\alpha} .
$$

The different equations of the model are summarized in Table 1.

Remark: In the present work, the coupling of damage and plasticity shares some similarities to the models in $[48,82]$, which do not consider strong coupling between damage and plasticity and are able to control the evolution of the plastic strain. In these models, the crack is driven only by the elastic strain energy as in (40). Here the evolution of plasticity delays the evolution of damage in some sense, unlike in the ductile models $[51,83]$ where the plasticity induces the evolution of fracture. Thus these models are proposed to simulate the brittle fracture in elastoplastic solids, and are well-suited for the simulation of PA12 material used in 3D printing.

\section{Construction of $\overline{\mathbf{C}}^{\alpha}(\alpha)$ using linear computational homogenization}

\subsection{Procedure}

We describe a procedure to evaluate numerically $\overline{\mathbf{C}}^{\alpha}(\alpha)$, which is the matrix form of $\overline{\mathbb{C}}^{\alpha}(\alpha)$ in (5), from preliminary calculations using linear computational homogenization (see e.g. handbooks in $[84,85]$ ). We consider the RVE (see Fig. 3 (b)) characterizing the layer material, which is defined in a domain $\omega \subset \mathbb{R}^{2}$. First, an interphase model must be defined. Here, we define this model as a geometric zone where the local elastic properties depend on $\alpha, \alpha \in[0 ; 1]$, such that $\mathbb{C}(\mathbf{x})=$ $g^{\alpha}(\alpha) \mathbb{C}^{0}(\mathbf{x})$. The function $g^{\alpha}(\alpha)$ is such that $g^{\alpha}(0)=1$ and $g^{\alpha}(1)=0$ and $\mathbb{C}^{0}(\mathbf{x})$ defines local undamaged elastic properties within the RVE. Then, for $\alpha=0$ the material is undamaged, while for $\alpha=1$ the local stiffness goes to zero. In the examples of Section 5 , this zone is taken as a layer 
with finite thickness, or the damaged zone obtained by a traction test on the RVE along $\mathbf{e}_{2}^{\prime}$. Then, for $K$ discrete values $\alpha^{n} \in\left[\alpha^{1}, \alpha^{2}, \ldots, \alpha^{K}\right]$, with $\alpha^{1}=0$ and $\alpha^{K}=1$ we apply the computational homogenization procedure described in the next section to determine the corresponding effective elasticity matrix $\overline{\mathbf{C}}^{\prime}\left(\alpha^{n}\right)$ in the basis $\left(\mathbf{e}_{1}^{\prime}, \mathbf{e}_{2}^{\prime}\right)$. A continuous representation of $\overline{\mathbf{C}}^{\prime}(\alpha)$ can then be obtained by fitting analytical functions to the values of $\overline{\mathbf{C}}^{\prime}\left(\alpha^{n}\right)$ or interpolating these discrete values. Finally, the effective elasticity matrix $\overline{\mathbf{C}}^{\alpha}(\alpha)$ in the Cartesian basis $\left(\mathbf{e}_{1}, \mathbf{e}_{2}\right)$ can be obtained by using a rotation relationship with respect to $\overline{\mathbf{C}}^{\prime}(\alpha)$, as described in the next section.

\subsection{Computational homogenization}

For each value $\alpha^{n}, n=1,2, \ldots, K$, the local elastic properties are considered as fixed $\mathbb{C}(\mathbf{x})=$ $\mathbb{C}\left(\mathbf{x}, \alpha^{n}\right) \forall \mathbf{x} \in \omega$. Then, the local problem to be solved over the RVE is as follows. Given $\mathbb{C}\left(\mathbf{x}, \alpha^{n}\right)$, $\bar{\varepsilon}$, where $\bar{\varepsilon}$ is a macroscopic strain, find $\varepsilon(\mathbf{x})$ such that :

$$
\begin{aligned}
& \nabla \cdot \boldsymbol{\sigma}(\mathbf{x})=\mathbf{0} \text { in } \omega, \\
& \sigma(\mathbf{x})=\mathbb{C}\left(\mathbf{x}, \alpha^{n}\right): \varepsilon(\mathbf{x}), \\
& \langle\varepsilon(\mathbf{x})\rangle=\bar{\varepsilon},
\end{aligned}
$$

where $\langle\rangle=.\frac{1}{V} \int_{V}(). d \Omega$ is the spatial averaging operator over $\omega$, and $V$ is the volume of $\omega$. (48) means that the RVE is subjected to a homogeneous strain $\bar{\varepsilon}$. This condition can be satisfied by prescribing appropriate boundary conditions over the RVE (see details in [84]), like e.g. the so-called periodic boundary conditions defined by:

$$
\mathbf{u}(\mathbf{x})=\bar{\varepsilon} \mathbf{x}+\tilde{\mathbf{u}}(\mathbf{x}) \quad \forall \mathbf{x} \in \partial \omega,
$$

where $\partial \omega$ is the boundary of $\omega$ and where $\tilde{\mathbf{u}}(\mathbf{x})$ is a periodic fluctuation over $\omega$. In practice, this condition is equivalent to impose the following constraint for two nodes of the FEM mesh on opposite sides of the boundary $\partial \omega$ of the RVE with coordinates $\mathbf{x}^{+}$and $\mathbf{x}^{-}$:

$$
\mathbf{u}\left(\mathbf{x}^{+}\right)-\mathbf{u}\left(\mathbf{x}^{-}\right)=\bar{\varepsilon}\left(\mathbf{x}^{+}-\mathbf{x}^{-}\right) .
$$

Details for implementing this condition by means of Lagrange multipliers within FEM discretizations of the RVE can be found in [84]. In (49),

$$
\bar{\varepsilon}=\frac{1}{2}\left(\mathbf{e}_{1}^{\prime} \otimes \mathbf{e}_{2}^{\prime}+\mathbf{e}_{2}^{\prime} \otimes \mathbf{e}_{1}^{\prime}\right) .
$$

The problem (46), (47) and (49) is then solved by FEM to obtain the displacement solutions $\mathbf{u}^{(11)}, \mathbf{u}^{(22)}, \mathbf{u}^{(12)}$ corresponding to the applied macroscopic strain fields

$$
\bar{\varepsilon}=\left[\begin{array}{lll}
1 & 0 & 0 \\
0 & 0 & 0 \\
0 & 0 & 0
\end{array}\right], \quad \bar{\varepsilon}=\left[\begin{array}{ccc}
0 & 0 & 0 \\
0 & 1 & 0 \\
0 & 0 & 0
\end{array}\right], \quad \bar{\varepsilon}=\left[\begin{array}{ccc}
0 & 1 / 2 & 0 \\
1 / 2 & 0 & 0 \\
0 & 0 & 0
\end{array}\right]
$$


expressed in the basis $\left(\mathbf{e}_{1}^{\prime}, \mathbf{e}_{2}^{\prime}\right)$. Then, due to the linearity of the problem, the displacement solution can be expressed as:

$$
\mathbf{u}(\mathbf{x})=\underbrace{\left[\begin{array}{lll}
\mathbf{u}^{(11)}(\mathbf{x}) & \mathbf{u}^{(22)}(\mathbf{x}) & \mathbf{u}^{(12)}(\mathbf{x})
\end{array}\right]}_{\mathbf{U}} \underbrace{\left[\begin{array}{c}
\bar{\varepsilon}_{11} \\
\bar{\varepsilon}_{22} \\
2 \bar{\varepsilon}_{12}
\end{array}\right]}_{[\bar{\epsilon}]},
$$

where $[\bar{\varepsilon}]$ is the macroscopic strain of the RVE in vector form. Then in each element, the stress is expressed as:

$$
[\boldsymbol{\sigma}(\mathbf{x})]=\mathbf{C}\left(\mathbf{x}, \alpha^{n}\right) \mathbf{B}(\mathbf{x}) \mathbf{U}^{e}[\bar{\varepsilon}],
$$

where $\mathbf{C}\left(\mathbf{x}, \alpha^{n}\right)$ is the local elasticity matrix associated to $\mathbb{C}\left(\mathbf{x}, \alpha^{n}\right), \mathbf{B}(\mathbf{x})$ is a matrix of displacement shape function derivatives, $\mathbf{U}^{e}$ is the element component of $\mathbf{U}$ and $[\boldsymbol{\sigma}(\mathbf{x})]$ is the vector form of stress tensor. Averaging over $\omega$, we have:

$$
[\overline{\boldsymbol{\sigma}}]=(\underbrace{\frac{1}{V} \int_{\omega} \mathbf{C}\left(\mathbf{x}, \alpha^{n}\right) \mathbf{B}(\mathbf{x}) \mathbf{U}^{e} d \Omega}_{\overline{\mathbf{C}}^{\prime}\left(\alpha^{n}\right)})[\bar{\varepsilon}],
$$

where

$$
\overline{\mathbf{C}}^{\prime}\left(\alpha^{n}\right)=\frac{1}{V} \int_{\omega} \mathbf{C}\left(\mathbf{x}, \alpha^{n}\right) \mathbf{B}(\mathbf{x}) \mathbf{U}^{e} d \Omega
$$

is the effective elasticity matrix with respect to the basis $\left(\mathbf{e}_{1}^{\prime}, \mathbf{e}_{2}^{\prime}\right)$. A continuous representation of $\overline{\mathbf{C}}^{\prime}(\alpha)$ can then be obtained by fitting analytical functions to the values of $\overline{\mathbf{C}}^{\prime}\left(\alpha^{n}\right)$ or interpolating these discrete values.

To express $\overline{\mathbf{C}}^{\alpha}$ in the Cartesian basis $\left(\mathbf{e}_{1}, \mathbf{e}_{2}\right)$, a rotation is applied through the relationship

$$
\bar{C}_{i j k l}^{\alpha}=R_{i p}(\theta) R_{j q}(\theta) R_{k r}(\theta) R_{l s}(\theta) \bar{C}_{p q r s}^{\prime},
$$

with

$$
\mathbf{R}(\theta)=\left[\begin{array}{ccc}
\cos (\theta) & -\sin (\theta) & 0 \\
\sin (\theta) & \cos (\theta) & 0 \\
0 & 0 & 1
\end{array}\right] .
$$

\section{Discretization and numerical implementation}

\subsection{Displacement problem}

We rewrite (31) as

$$
\mathbf{R}=\int_{\Omega} \boldsymbol{\sigma}: \boldsymbol{\varepsilon}^{e}(\delta \mathbf{u}) d \Omega-\int_{\partial \Omega_{F}} \overline{\mathbf{F}} \cdot \delta \mathbf{u} d S=0 .
$$


In a standard Newton method, the displacements are updated for each loading increment by solving the tangent problem:

$$
D_{\Delta u} \mathbf{R}\left(\mathbf{u}^{(k)}\right)=-\mathbf{R}\left(\mathbf{u}^{(k)}\right)=0,
$$

where $D_{\mathbf{v}}($.$) has been defined in (16), and \mathbf{u}^{(k)}$ is the displacement solution known from the previous iteration. The displacement corrections are obtained as

$$
\mathbf{u}^{(k+1)}=\mathbf{u}^{(k)}+\Delta \mathbf{u} .
$$

In $(60)$,

$$
D_{\Delta \mathbf{u}} \mathbf{R}\left(\mathbf{u}^{(k)}\right)=\int_{\Omega} \frac{\partial \boldsymbol{\sigma}}{\partial \varepsilon^{e}}: \varepsilon^{e}(\Delta \varepsilon): \varepsilon^{e}(\delta \varepsilon) d \Omega,
$$

with

$$
\frac{\partial \boldsymbol{\sigma}}{\partial \varepsilon^{e}}=\mathbb{C}_{s}(\mathbf{u}, d, \alpha)
$$

We can obtain the FEM approximations as:

$$
\begin{aligned}
& \mathbf{u}=\mathbf{N}_{\mathbf{u}} \mathbf{u}^{e}, \quad \delta \mathbf{u}=\mathbf{N}_{\mathbf{u}} \delta \mathbf{u}^{e}, \quad \Delta \mathbf{u}=\mathbf{N}_{\mathbf{u}} \Delta \mathbf{u}^{e}, \\
& {[\varepsilon](\mathbf{u})=\mathbf{B}_{\mathbf{u}} \mathbf{u}^{e}, \quad\left[\varepsilon^{e}\right](\Delta \mathbf{u})=\mathbf{B}_{\mathbf{u}} \Delta \mathbf{u}^{e}, \quad\left[\varepsilon^{e}\right](\delta \mathbf{u})=\mathbf{B}_{\mathbf{u}} \delta \mathbf{u}^{e},}
\end{aligned}
$$

where $\mathbf{N}_{\mathbf{u}}$ and $\mathbf{B}_{\mathbf{u}}$ are respectively matrices of displacement shape function and its derivative, $\mathbf{u}^{e}$, $\delta \mathbf{u}^{e}$ and $\Delta \mathbf{u}^{e}$ are nodal displacement components in one element, nodal trial function components and nodal incremental displacement components, respectively.

After discretization, the linear system (60) with the displacement corrections (61) reduces to a standard Newton-type iteration:

$$
\begin{aligned}
& \mathbf{K}_{\tan } \Delta \mathbf{u}=-\mathbf{R}\left(\mathbf{u}^{(k)}\right), \quad \mathbf{u}^{(k+1)}=\mathbf{u}^{(k)}+\Delta \mathbf{u}, \\
& \mathbf{K}_{\tan }=\int_{\Omega} \mathbf{B}_{\mathbf{u}}^{T} \mathbf{C}_{s} \mathbf{B}_{\mathbf{u}} d \Omega,
\end{aligned}
$$

and

$$
\mathbf{R}\left(\mathbf{u}^{(k)}\right)=\int_{\Omega} \mathbf{B}_{\mathbf{u}}^{T} \boldsymbol{\sigma}^{(k)} d \Omega-\int_{\partial \Omega_{F}} \mathbf{N}_{\mathbf{u}}^{T} \overline{\mathbf{F}} d S
$$

and where $\mathbf{C}_{s}$ is the matrix form corresponding to the fourth-order elastoplastic consistent tangent operator $\mathbb{C}_{s}$ in (63), which is determined by the classical elastic predictor and plastic corrector (return-mapping) algorithm outlined in [86]. The iterative update (65) is performed until convergence is achieved in the sense $\|\Delta \mathbf{u}\| /\left\|\mathbf{u}^{(k+1)}-\mathbf{u}^{(0)}\right\| \leq$ tol, with tol a numerical tolerance parameter. 


\subsection{Bulk damage problem}

The damages and damage gradients are approximated in one element by

$$
d=\mathbf{N d}^{e}, \quad \nabla d=\mathbf{B d}^{e}, \quad \alpha=\mathbf{N} \boldsymbol{\alpha}^{e}, \quad \nabla \alpha=\mathbf{B} \boldsymbol{\alpha}^{e},
$$

where $\mathbf{N}$ and $\mathbf{B}$ are matrices of damage shape function and of damage shape function derivative, respectively, $\mathbf{d}^{e}$ and $\boldsymbol{\alpha}^{e}$ denote respectively nodal bulk and interfacial damage in one element.

The discretization of bulk damage problem in (39) results into the following discrete system of equations:

$$
\mathbf{K}_{d} \mathbf{d}=\mathbf{F}_{d}
$$

in which

$$
\mathbf{K}_{d}=\int_{\Omega}\left[\left(2 \mathcal{H}+\frac{g_{c}^{d}}{\ell_{d}}\right) \mathbf{N}^{T} \mathbf{N}+g_{c}^{d} \ell_{d} \mathbf{B}^{T} \mathbf{B}\right] d \Omega
$$

and

$$
\mathbf{F}_{d}=\int_{\Omega} 2 \mathbf{N}^{T} \mathcal{H} d \Omega,
$$

where $\mathcal{H}$ is given in (40).

\subsection{Interfacial damage problem}

The discretization of interfacial damage problem in (43) results into the following discrete system of equations:

$$
\mathbf{K}_{\alpha} \boldsymbol{\alpha}+\mathbf{F}_{\alpha}(\boldsymbol{\alpha})=\mathbf{0}
$$

in which

$$
\mathbf{K}_{\alpha}=\int_{\Omega}\left(\frac{g_{c}^{\alpha}}{\ell_{\alpha}} \mathbf{N}^{T} \mathbf{N}+g_{c}^{\alpha} \ell_{\alpha} \mathbf{B}^{T} \boldsymbol{\omega}^{\alpha} \mathbf{B}\right) d \Omega
$$

and

$$
\mathbf{F}_{\alpha}(\boldsymbol{\alpha})=\int_{\Omega} \frac{1}{2} g(d) \varepsilon^{e}: \frac{\partial \overline{\mathbb{C}}^{\alpha}}{\partial \alpha}: \varepsilon^{e} \mathbf{N}^{T} d \Omega .
$$

Note that in general, (72) is a nonlinear problem and must be solved through a standard Newton-type iteration as used in Section 4.1. In Section 5.1, we will choose a quadratic model for the components $\overline{\mathbf{C}}^{\alpha}(\alpha)$ to keep (72) as linear.

\subsection{Numerical implementation}

In the present work, a single-step staggered scheme based on small load increments is employed, where at each load increment the displacement problem is solved for fixed bulk and interfacial damage fields which are known from the previous time step. The bulk damage problem is then solved with the new displacement field and previous interfacial damage field. Finally, the interfacial damage problem is solved with the new displacement field and bulk damage field. 

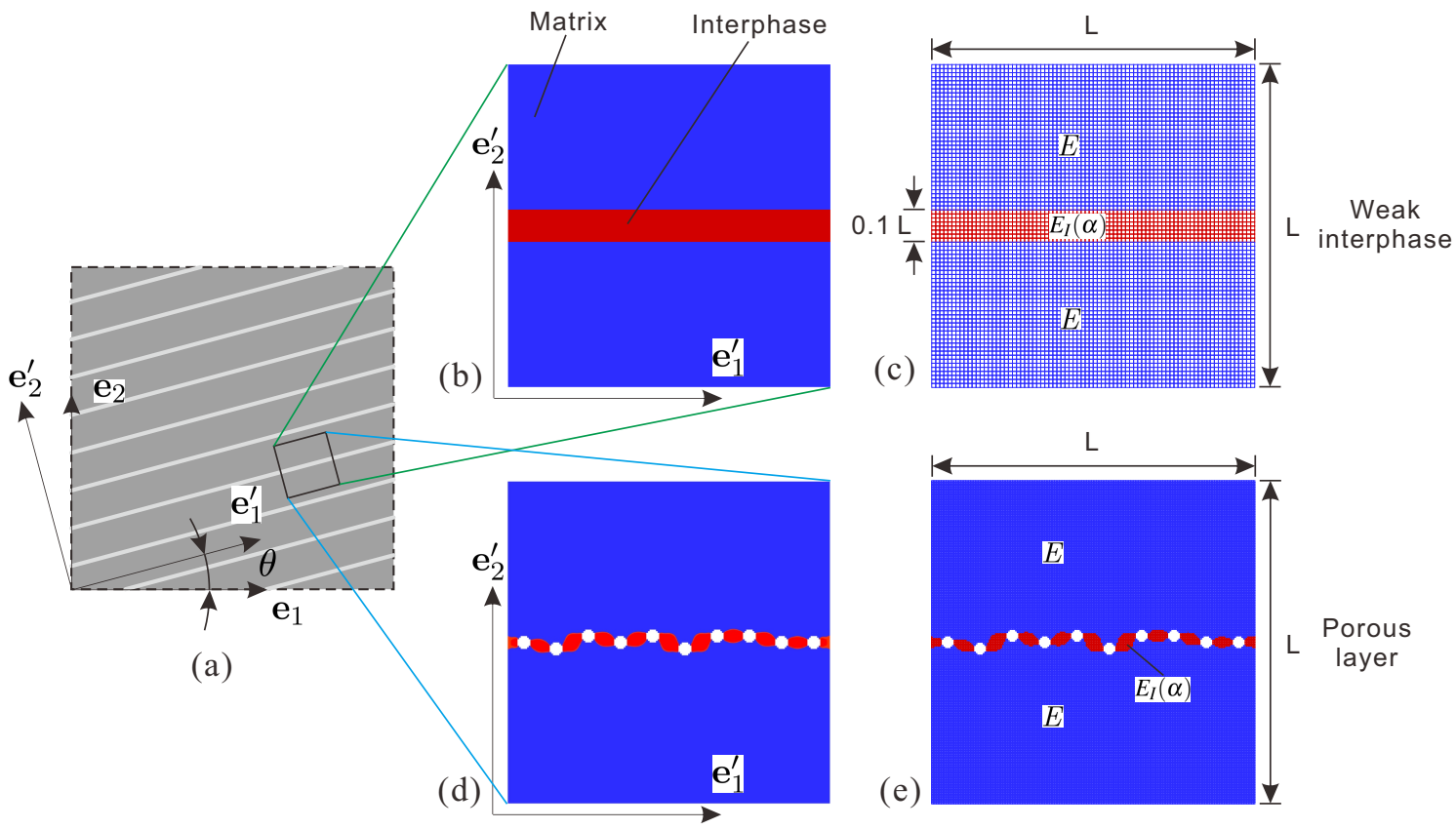

Figure 4: (a) 3D printed material with a layered microstructure; (b) RVE with a straight interphase; (c) geometry and corresponding mesh; (d) RVE with a porous interphase and (e) geometry and corresponding mesh. $L=1 \mathrm{~mm}$.

\section{Numerical examples}

We consider a layered material (see Fig. 4 (a)), where the layers have an angle $\theta$ with respect to the main frame $\left(\mathbf{e}_{1}, \mathbf{e}_{2}\right)$.

\subsection{Identification of $\overline{\mathbf{C}}^{\alpha}$}

In this example, we apply the procedure described in Section 3 for the identification of $\overline{\mathbf{C}}^{\alpha}$ for two different RVEs: one implying a straight interphase (Fig. 4(b)) and one with an irregular distribution of holes along one direction, possibly characterizing a porous interphase induced during a 3D printing SLS process (Fig. 4(d)).

\subsubsection{Layered structure}

We first consider the RVE described in Fig. 4(b), which consists of a square domain of length $L=1 \mathrm{~mm}$ containing a rectangular interphase with thickness $0.1 L$ in the middle of the RVE and parallel to $\mathbf{e}_{1}^{\prime}$. The corresponding mesh is described in Fig. 4(c), and consists into $80 \times 80$ square 4 -node elements. The blue mesh corresponds to the layers and the red mesh corresponds to an interphase with weaker properties. Both materials (matrix and interphase, see Fig. 4) are assumed to be isotropic-elastoplastic with linear hardening. The material parameters for the matrix are chosen as follows (unless otherwise stated): $E=10 \mathrm{GPa}, \nu=0.25, \sigma_{y}=0.08 \mathrm{GPa}$ and $H=0.1$ GPa. In (12), $\xi^{\alpha}=30$ is used. We denote the Young's modulus of the interphase $E_{I}$ with respect to the micro interfacial damage parameter $\alpha$ according to:

$$
E_{I}(\alpha)=G(\alpha) E, \quad G(\alpha)=\frac{(1-\alpha)^{2}}{\chi-(\chi-1)(1-\alpha)^{2}} \quad \text { with } \quad \chi \geq 1
$$




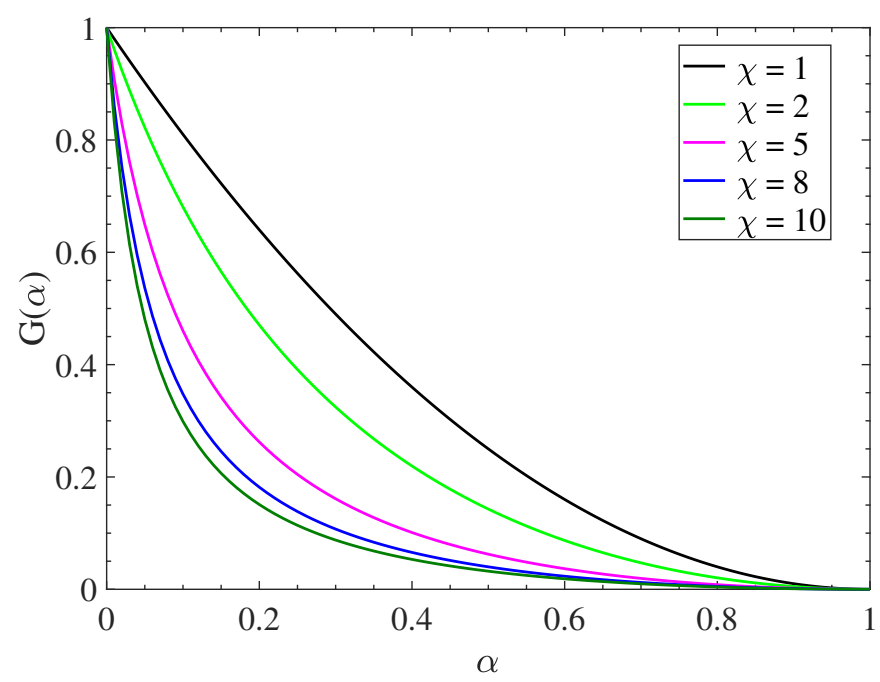

Figure 5: The degradation function $G(\alpha)$ for various parameter $\chi$.

where the degradation function $G(\alpha)$ is taken from [51]. Fig. 5 shows the sensitivity of $G(\alpha)$ with respect to $\chi$. The Poisson's ratio $\nu_{I}$ for the interphase is assumed to be independent on $\alpha: \nu_{I}=\nu$.

Using the plane stress assumption, the elasticity matrix for the interphase material can be expressed as

$$
\mathbf{C}_{I}(\alpha)=\frac{E_{I}(\alpha)}{1-\nu_{I}^{2}}\left[\begin{array}{ccc}
1 & \nu_{I} & 0 \\
\nu_{I} & 1 & 0 \\
0 & 0 & \frac{1-\nu_{I}}{2}
\end{array}\right]
$$

As mentioned above, to maintain the interfacial damage problem in (72) related to $\alpha$ as linear, we introduce a quadratic fit of the different numerical components of $\overline{\mathbf{C}}^{\prime}$. The effective elasticity matrix is computed by the procedure in Section 3. The evolution of the different effective components are depicted in Fig. 6. It can be seen from this figure that the effective elasticity components $\bar{C}_{12}^{\prime}, \bar{C}_{22}^{\prime}$ and $\bar{C}_{33}^{\prime}$ decrease to zero when $\alpha=1$, and $\bar{C}_{11}^{\prime}$ decreases slightly to a nonzero constant when $\alpha=1$.

To ensure that (i) (72) remains linear and that (ii) $0 \leq \alpha \leq 1$ when solving (72), a quadratic function :

$$
\bar{C}_{i j}^{\prime}(\alpha)=a_{1}^{i j}+a_{2}^{i j}(1-\alpha)^{2}
$$

is employed to fit the evolution of the effective elastic components which are thought as a function of $\alpha$, as shown in Fig. 6, we obtain $\overline{\mathbf{C}}^{\prime}$ as

$$
\overline{\mathbf{C}}^{\prime}(\alpha)=\left[\begin{array}{ccc}
8.999+1.668(1-\alpha)^{2} & 2.667(1-\alpha)^{2} & 0 \\
2.667(1-\alpha)^{2} & 10.667(1-\alpha)^{2} & 0 \\
0 & 0 & 4(1-\alpha)^{2}
\end{array}\right] .
$$




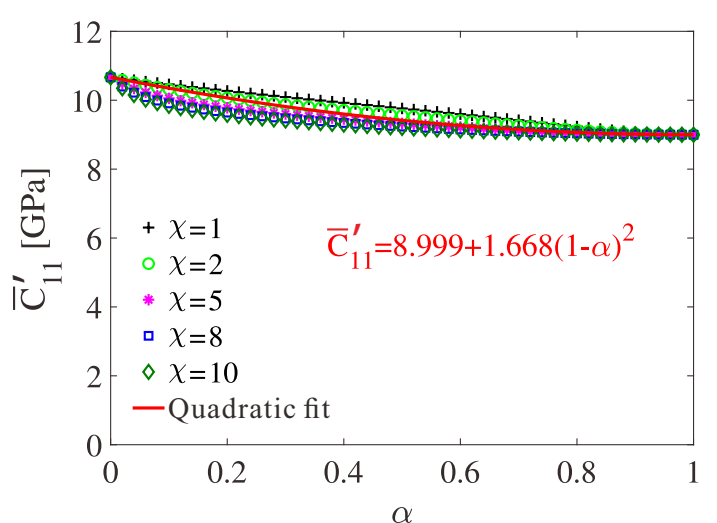

(a)

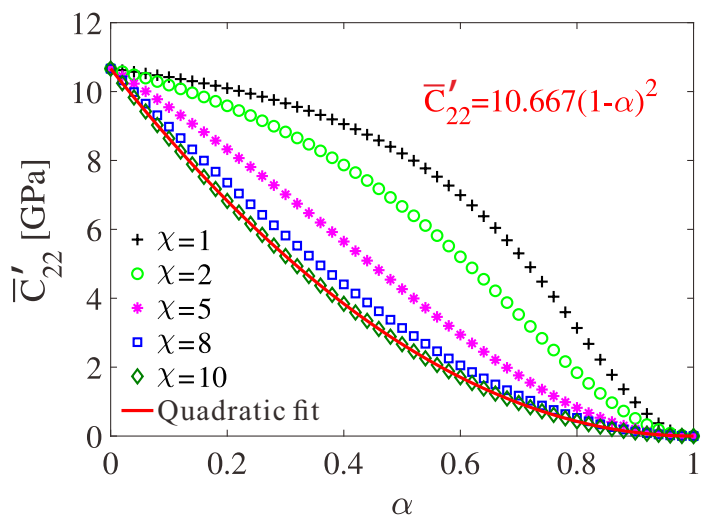

(c)

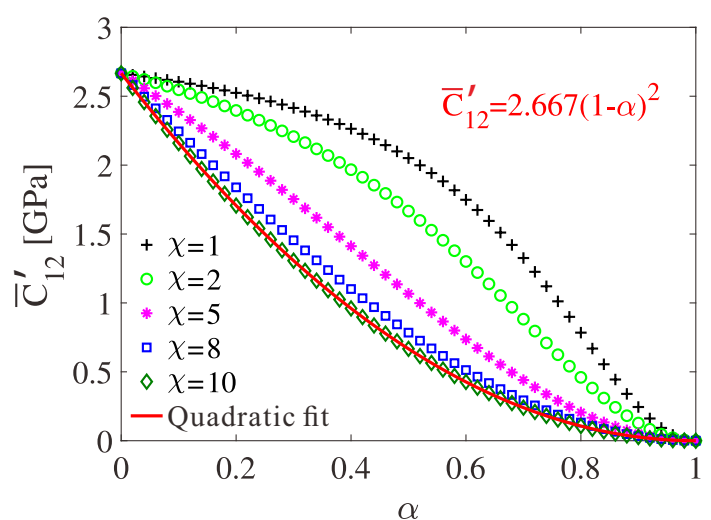

(b)

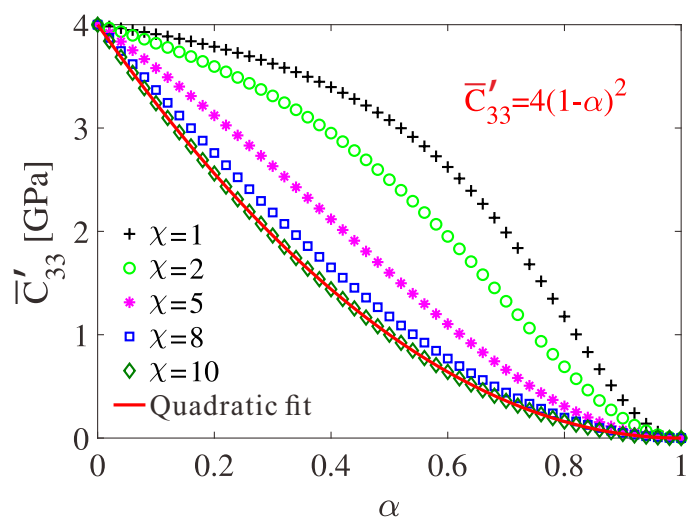

(d)

Figure 6: Layered structure: evolution of different effective components with respect to the interfacial damage $\alpha$. 
Then we can obtain $\overline{\mathbf{C}}^{\alpha}$ by using the rotation relationship defined in (57).

We can note in Fig. 6 that in the case $\chi=10$, the quadratic fit provides a good approximation of the discrete values of $\overline{\mathbf{C}}^{\prime}\left(\alpha^{n}\right)$. It is not the case for the other values of $\chi$ : then, higher order functions might be used to fit the numerical curves. However, this will lose the linear character of the interfacial damage problem in (72) and numerical constraints will be required to prescribe $0 \leq \alpha \leq 1$. The study of such case may require additional developments and is reported to future studies.

\subsubsection{Porous layer}

In the next example, we identify $\overline{\mathbf{C}}^{\alpha}$ for the RVE including an irregular distribution of pores along $\mathbf{e}_{1}^{\prime}$ (see Fig. 4 (d)). The corresponding mesh is depicted in Fig. 4(e), where the RVE domain is uniformly discretized into $200 \times 200$ elements and the elements at the position of the pores are removed. Here, we create a domain with the properties depending on $\alpha$ as follows. We first conduct a phase field analysis applying $\bar{\varepsilon}=\bar{\varepsilon}_{22} \mathbf{e}_{2}^{\prime} \otimes \mathbf{e}_{2}^{\prime}$ as described in (50). Then, we select the elements where $d>0.9$ as belonging to the domain where the properties are the one of the interphase in the previous section. In Fig. 4(e), these elements are depicted in red color. Here, a quadratic fit is employed for $\chi=20$.

The evolution of the different effective components of $\overline{\mathbf{C}}^{\prime}$ are depicted in Fig. 7, and are obtained as:

$$
\overline{\mathbf{C}}^{\prime}(\alpha)=\left[\begin{array}{ccc}
9.486+0.85(1-\alpha)^{2} & 2.575(1-\alpha)^{2} & 0 \\
2.575(1-\alpha)^{2} & 10.231(1-\alpha)^{2} & 0 \\
0 & 0 & 3.802(1-\alpha)^{2}
\end{array}\right]
$$

Similarly, $\overline{\mathbf{C}}^{\alpha}$ for porous layer RVE can be obtained using (57).

We can observe similar results as compared to the results of Fig. 6, except that the initial stiffness matrix at $\alpha=0$ is different and slightly anisotropic $\left(\bar{C}_{1111}^{\prime}(\alpha=0) \neq \bar{C}_{2222}^{\prime}(\alpha=0)\right)$ here because of the presence of pores along $\mathbf{e}_{1}^{\prime}$.

\subsection{One-notch square plate}

In this example, we investigate the proposed model with a one-notch square plate, as shown in Fig. 8. The boundary conditions are as follows: on the lower end $(y=0)$, the $y$-displacement are fixed, while the $x$-displacement are free and the node $(x=0, y=0)$ is fixed. On the upper end, the $x$-displacement are free, while the $y$-displacement are prescribed to an increasing value of $\bar{U}$ with $\Delta \bar{U}=5 \times 10^{-4} \mathrm{~mm}$ during the simulation. The fracture toughness for bulk fracture and interfacial damage are $g_{c}^{d}=4 \times 10^{-3} \mathrm{kN} / \mathrm{mm}$ and $g_{c}^{\alpha}=1 \times 10^{-3} \mathrm{kN} / \mathrm{mm}$, respectively. The length scale parameters for bulk damage and interfacial damage are $\ell_{d}=\ell_{\alpha}=0.2 \mathrm{~mm}$. The values of $\overline{\mathbf{C}}^{\alpha}$ have been obtained by using the interphase RVE. The mesh is composed of 10050 4-node regular elements, as shown in Fig. 8 (b).

Fig. 9 shows the load-displacement curves obtained from different layer orientations, using both RVEs. We can note that the curves for $\theta=0^{\circ}$ and $\theta=30^{\circ}$ are very close, while for $\theta=60^{\circ}$ a higher peak load is noticed. A significant plastic response is only noticed for $\theta=90^{\circ}$. We can also note that the response obtained through both RVEs is not significantly different.

As the responses of the structures for both RVE models in Fig. 9 are close, we only consider the model using the interphase RVE in the next examples. 


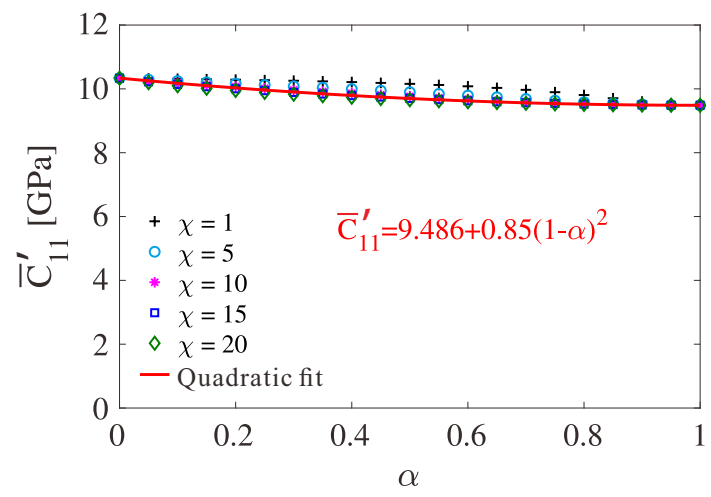

(a)

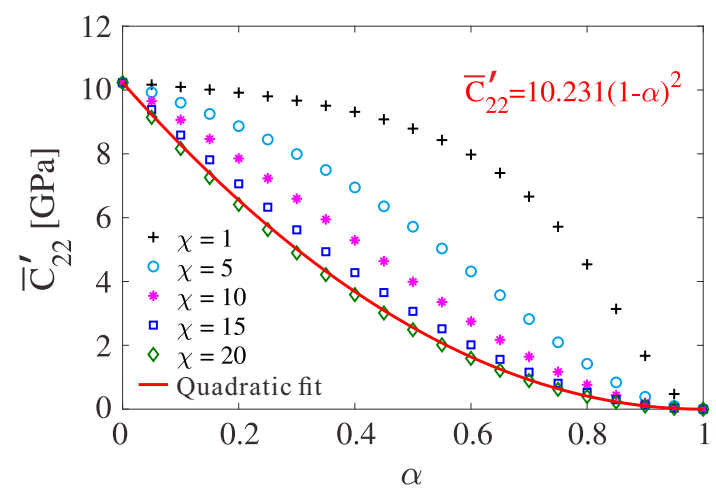

(c)

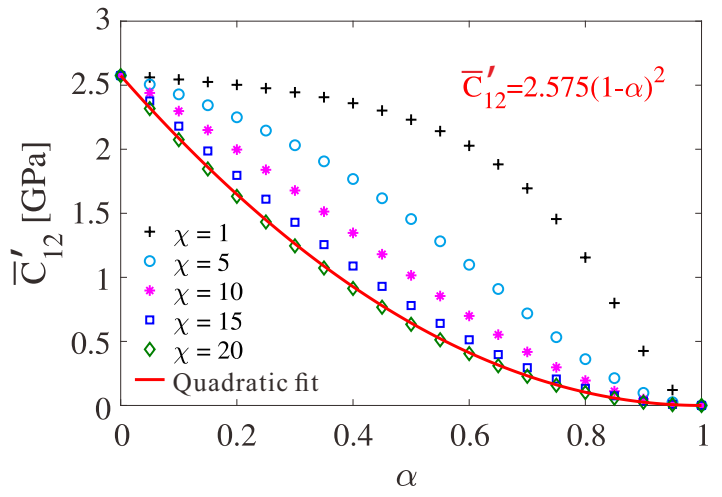

(b)

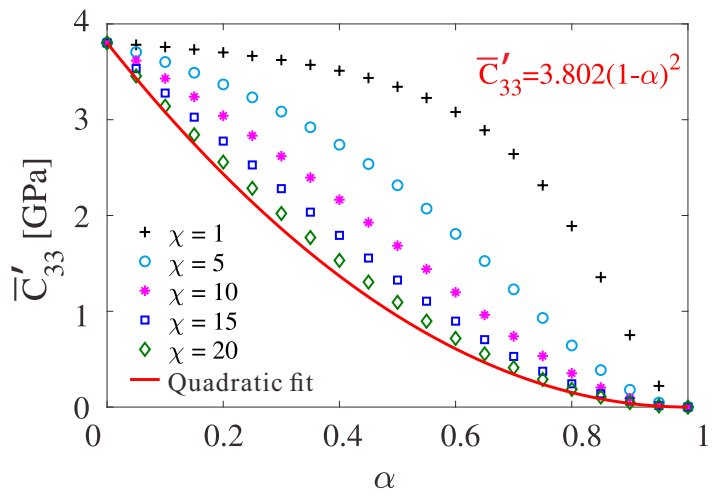

(d)

Figure 7: Porous layer: evolution of different effective components with respect to the interfacial damage $\alpha$.

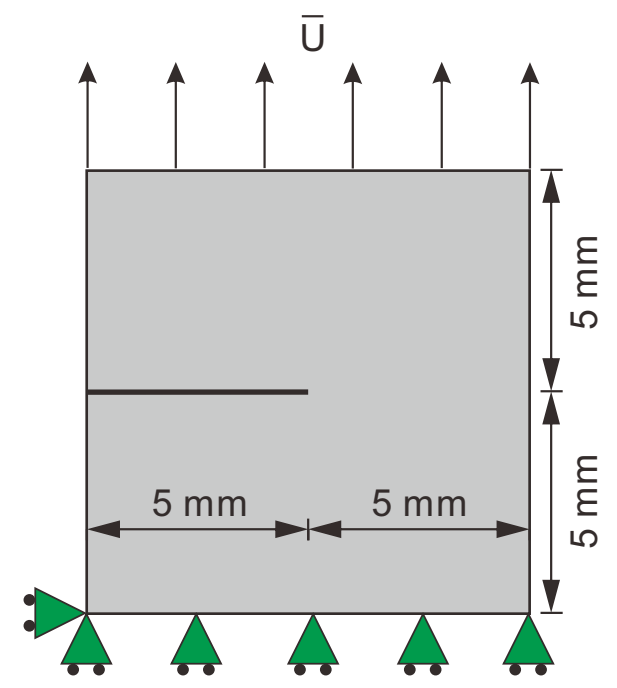

(a)

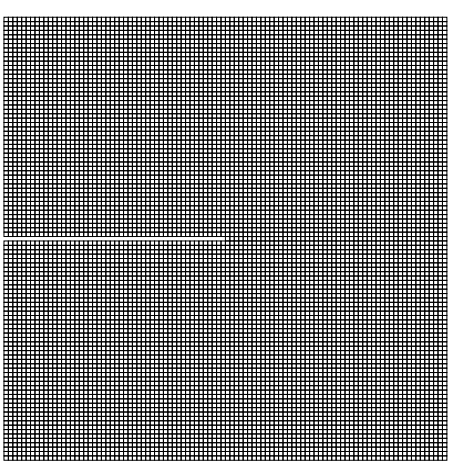

(b)

Figure 8: One-notch square plate: (a) geometry and boundary conditions; (b) finite element model. 


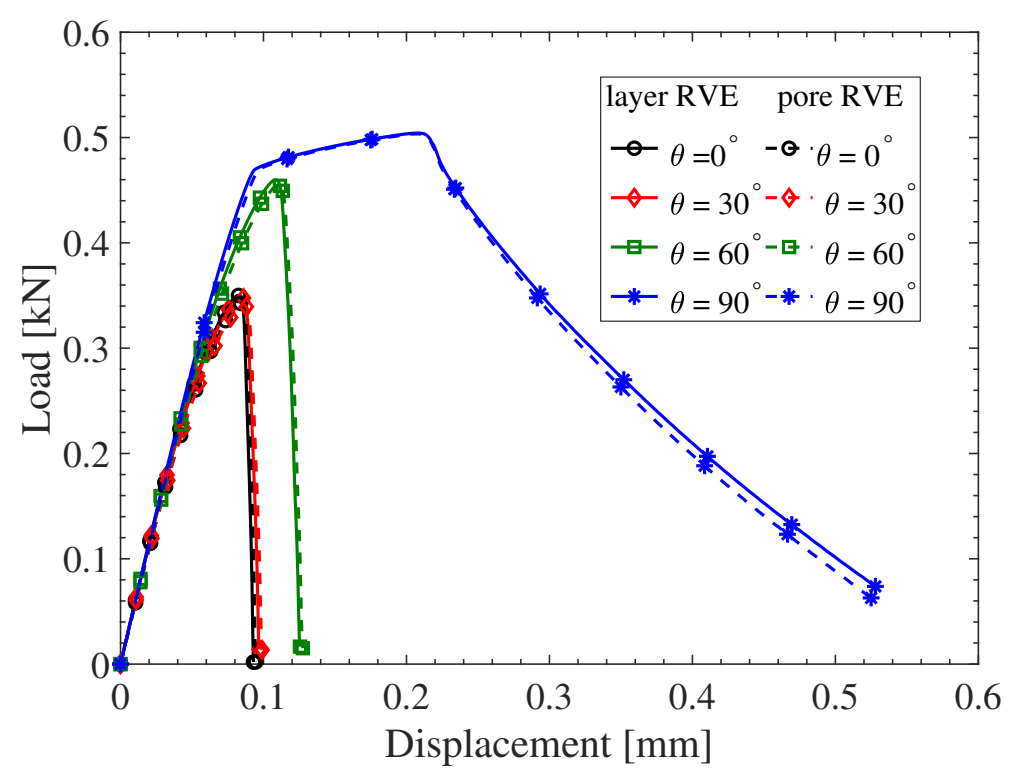

Figure 9: One-notch square plate. Load-displacement curves for different layer orientations, obtained from (a) $\overline{\mathbf{C}}_{\alpha}$ in (78) and (b) $\mathbf{C}_{\alpha}$ in (79).

Fig. 10 shows the final interfacial damage, bulk crack and equivalent plastic strain field for different layer orientations, using the interphase RVE. For $\theta=0^{\circ}, \theta=30^{\circ}$, and $\theta=60^{\circ}$, the directions of micro interfacial damage $\alpha$ correspond to the layer directions. For $\theta=90^{\circ}$, after a long plastic stage, the layer damage $d$ is perpendicular to the layer orientation. We can also observe a significant distribution of the equivalent plastic strain field in that case, which does not occur for the other orientations.

Then, the present model is able to describe the transition from quasi-brittle behavior to elastoplastic behavior for 3D printed materials with respect to the layer orientations.

Next, we test the convergence of the model with respect to the mesh refinement. For this purpose, the previous test is conducted for $\theta=90^{\circ}$ with three refined meshes with 3320 elements, 7076 and 14,818 elements, respectively. All meshes include a refined region where the crack is expected to propagate, as shown in Fig. 11, which shows the final bulk damage fields for the 3 meshes. The load-displacement curves for the 3 meshes are provided in Fig. 12, which demonstrates the convergence of the method with mesh refinement.

\subsection{Two-notch specimen}

In this next example, we investigate the proposed model on a two-notch specimen, as shown in Fig. 13(a). The boundary conditions are identical as in the example of Section 5.2. External loading is applied by displacement control through a series of load increments with a fixed step value $\Delta \bar{U}=1 \times 10^{-3} \mathrm{~mm}$. The spatial discretization of the model comprises 20,777 4-node quadrilateral elements, with refinement in the central region where the crack is expected to propagate (see Fig. 13(b)). The fracture toughness for bulk layer fracture and micro interfacial damage are $g_{c}^{d}=6.5 \times 10^{-3} \mathrm{kN} / \mathrm{mm}$ and $g_{c}^{\alpha}=1.5 \times 10^{-3} \mathrm{kN} / \mathrm{mm}$, respectively. The length scale parameters are chosen as $\ell_{d}=\ell_{\alpha}=0.4 \mathrm{~mm}$. 

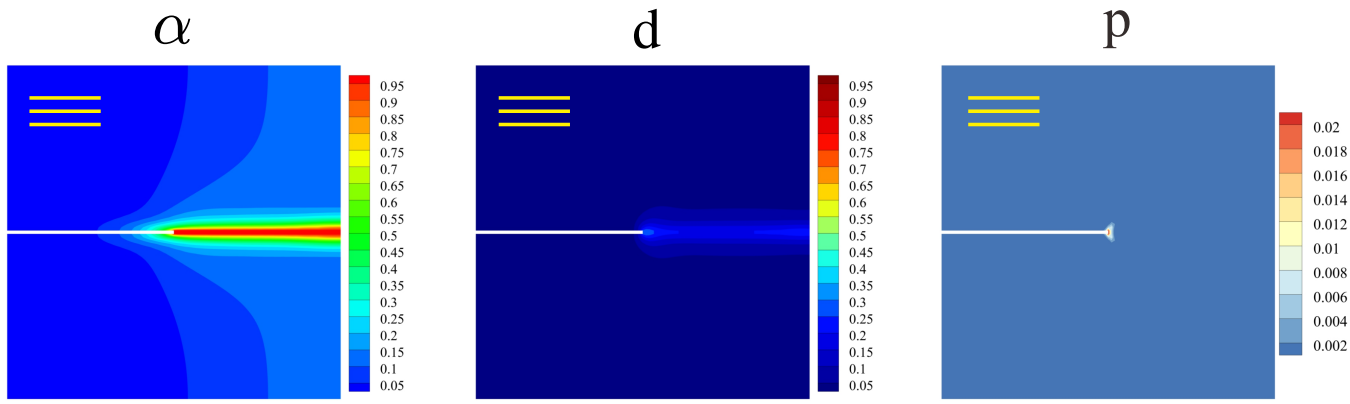

(a) $\theta=0^{\circ}$
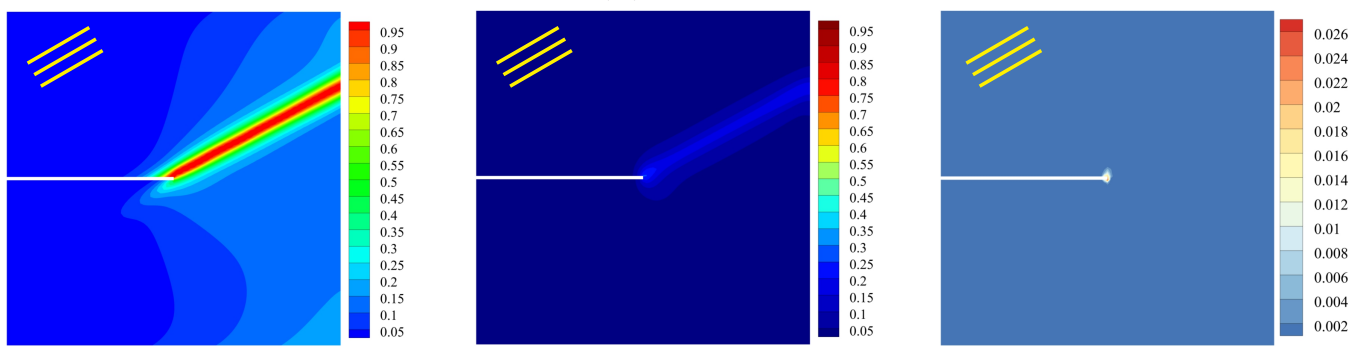

(b) $\theta=30^{\circ}$
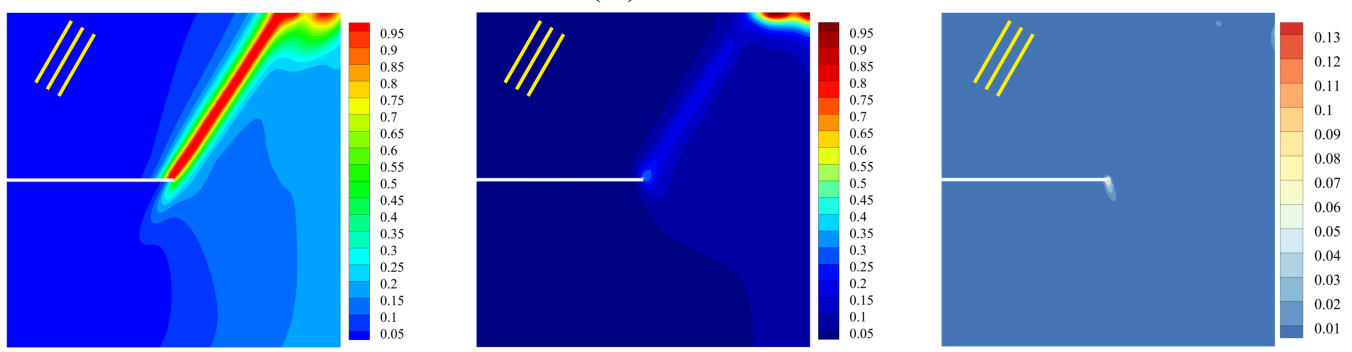

(c) $\theta=60^{\circ}$
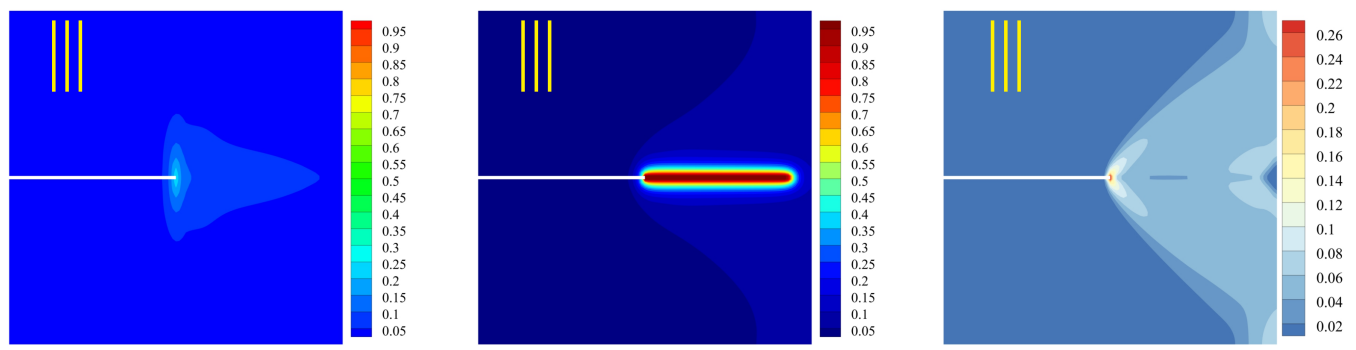

(d) $\theta=90^{\circ}$

Figure 10: One-notch square plate. Final interfacial damage, bulk crack and equivalent plastic strain field for different layer orientations. 

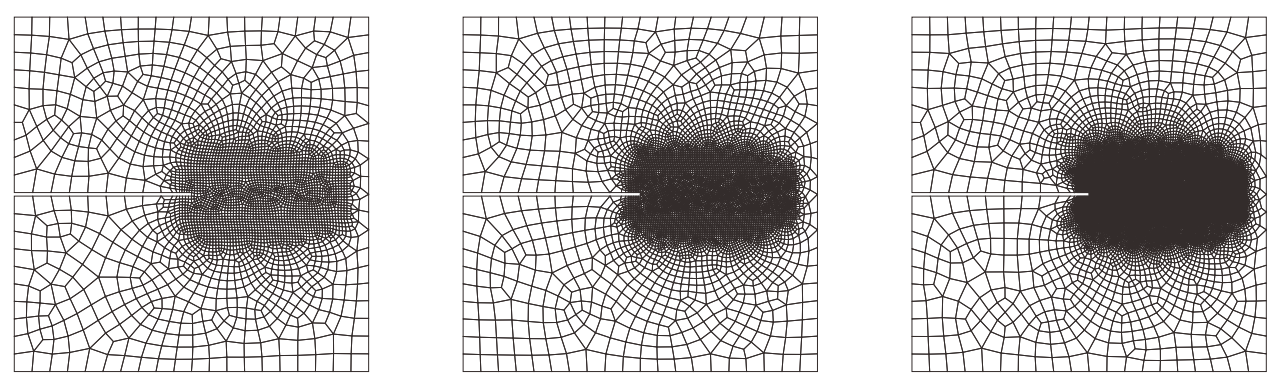

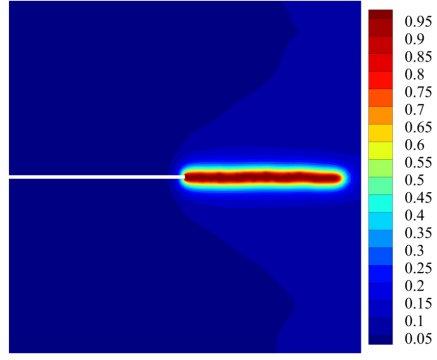

(a)

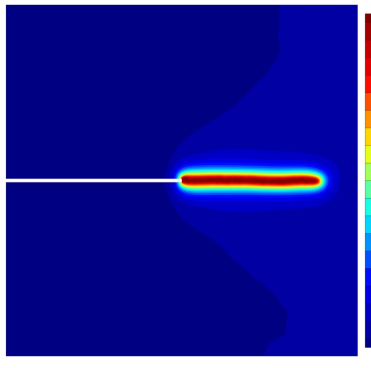

(b)

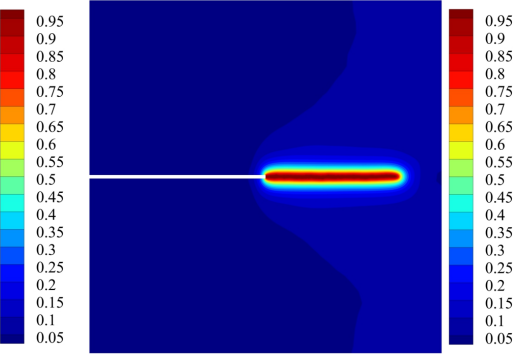

(c)

Figure 11: Meshes and final bulk layer damage fields $d(\mathbf{x})$ for $\theta=90^{\circ}$ : (a) coarse mesh (3320 elements), (b) medium mesh (7076 elements) and (c) fine mesh (14,818 elements).

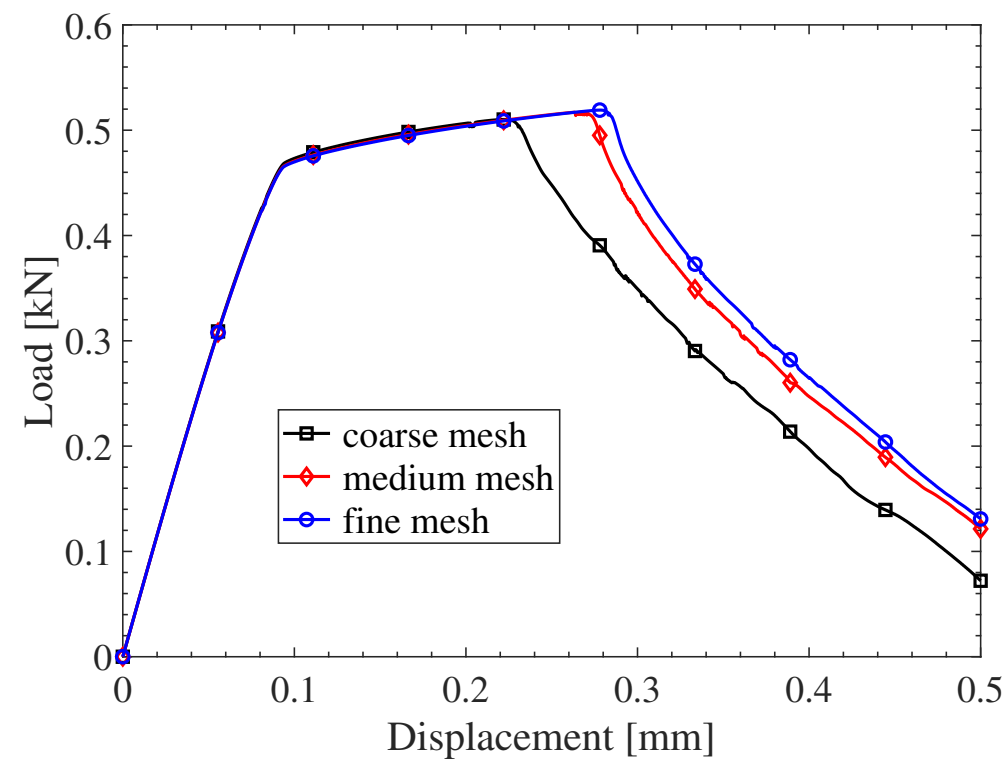

Figure 12: Load-displacement curves for three finite element meshes, one-notch plate example. 


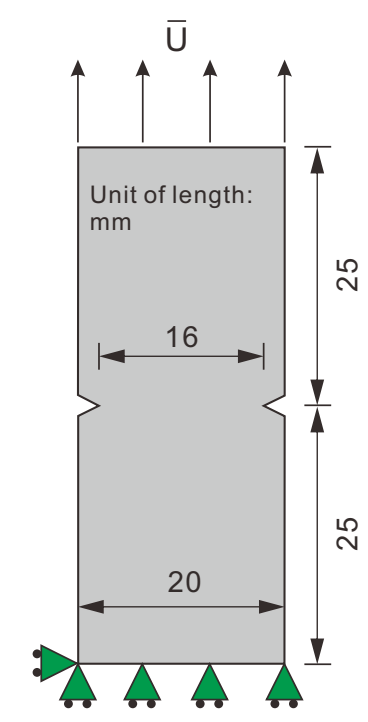

(a)

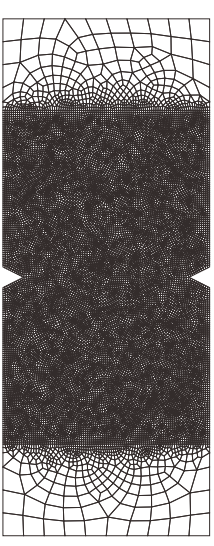

(b)

Figure 13: Two-notch specimen: (a) geometry and boundary conditions; (b) finite element model.

The load-displacement curves for the different layer orientations are shown in Fig. 14. We can see that the peak load increases with an increase of the layer orientation angle $\theta$, which matches the experimental tendency observed in the experimental results of [12]. Here again, only the curve for $\theta=90^{\circ}$ exhibits a significant plastic stage. Fig. 15 shows the final micro interfacial damage, bulk layer damage and equivalent plastic strain field for different layer directions. We can observe that when $\theta=0^{\circ}, \theta=30^{\circ}$ and $\theta=60^{\circ}$, the failure of the structure is mainly caused by the evolution of the micro interfacial damage $\alpha$, while for $\theta=90^{\circ}$ the bulk crack $d$ perpendicular to the layer direction causes the final failure of the structure. This example further illustrates that the proposed model can simulate the different fracture resistance caused by the different layer orientations.

\subsection{Two-hole specimen}

In the next example, a structure with two asymmetric holes, as shown in Fig. 16(a), is investigated. The boundary conditions are similar as the example of Section 5.3. The spatial discretization of the model comprises 13,839 4-node quadrilateral elements, with refinement around holes where the crack is expected to propagate, see Fig. 16(b). The fracture toughness for bulk layer damage $d$ and micro interfacial damage $\alpha$ are $g_{c}^{d}=6 \times 10^{-3} \mathrm{kN} / \mathrm{mm}$ and $g_{c}^{\alpha}=1.5 \times 10^{-3} \mathrm{kN} / \mathrm{mm}$, respectively. The length scale parameters are $\ell_{d}=\ell_{\alpha}=0.4 \mathrm{~mm}$.

Fig. 17 shows the load-displacement curves for different layer orientations. As in previous examples, quasi-brittle behavior is noticed for $\theta=0^{\circ}, \theta=30^{\circ}$ and $\theta=60^{\circ}$, while a plastic stage is observed before the final failure of the structure for $\theta=90^{\circ}$. Fig. 18 shows the final micro interfacial damage, bulk layer damage and equivalent plastic strain field for different layer orientations. We can note that the presence of the holes induces a more complex local strain state, which leads to both micro interfacial and layer bulk damage for $\theta=60^{\circ}$ and $\theta=90^{\circ}$. This example further demonstrates that the proposed model allows describing a complex anisotropic damage phenomenon combining interfacial damage and bulk crack in complex configurations. 


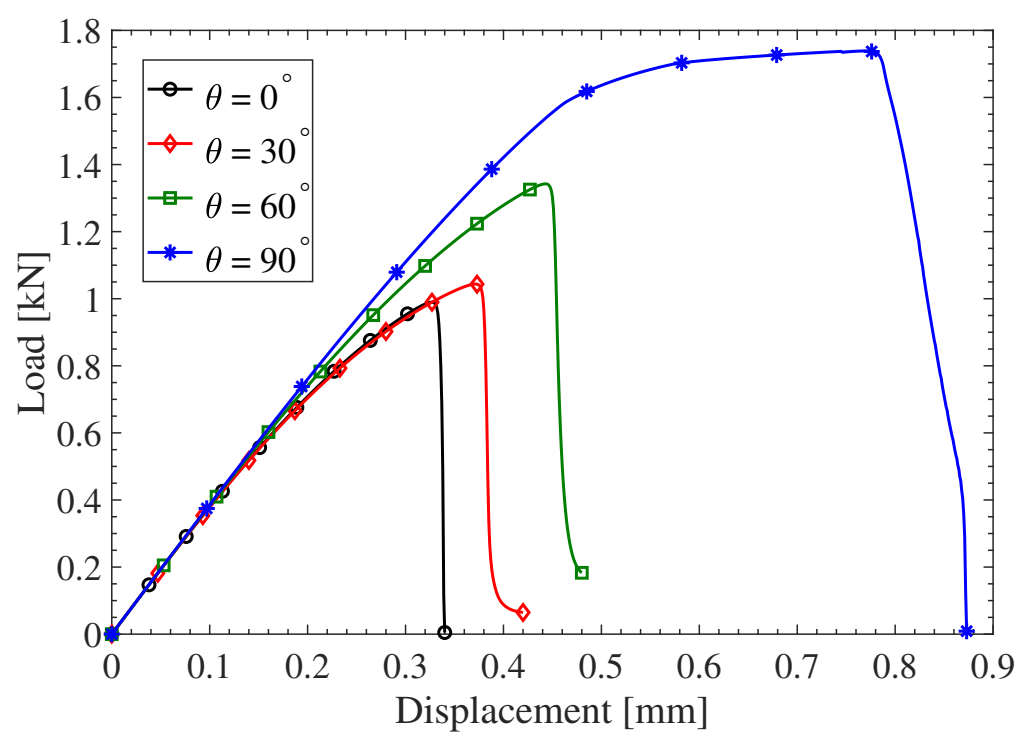

Figure 14: Two-notch specimen. Load-displacement curves for different layer orientations.

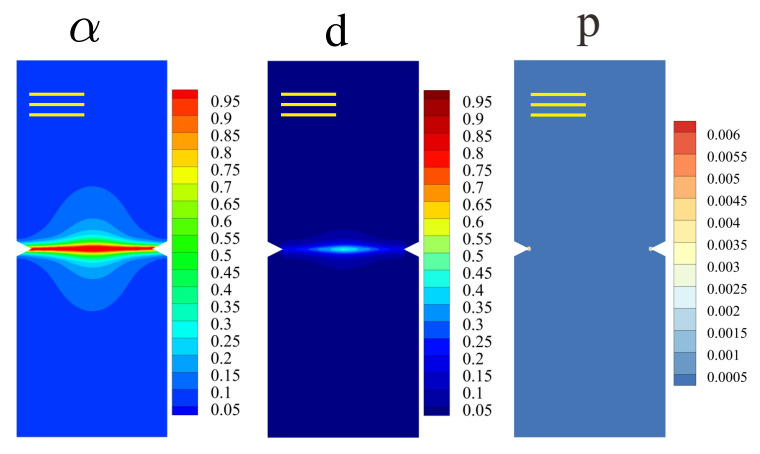

(a) $\theta=0^{\circ}$
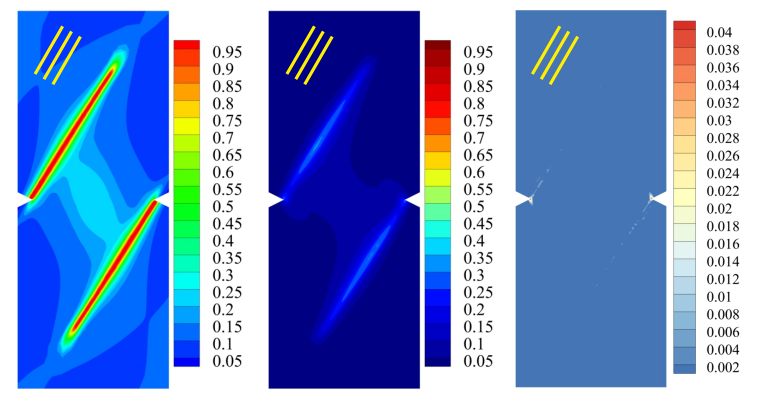

(c) $\theta=60^{\circ}$

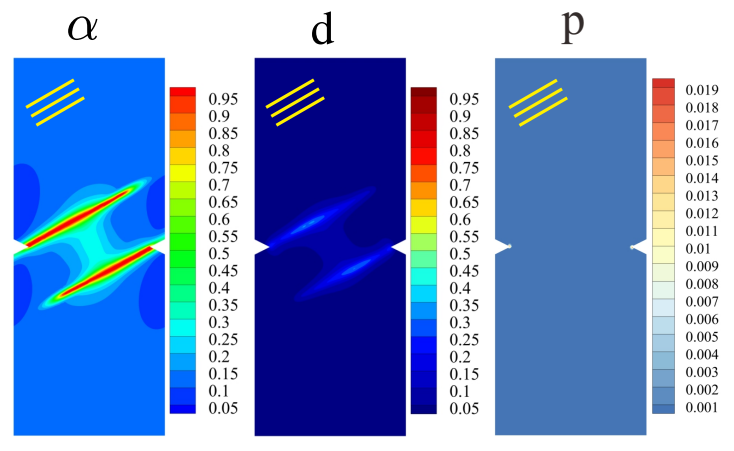

(b) $\theta=30^{\circ}$

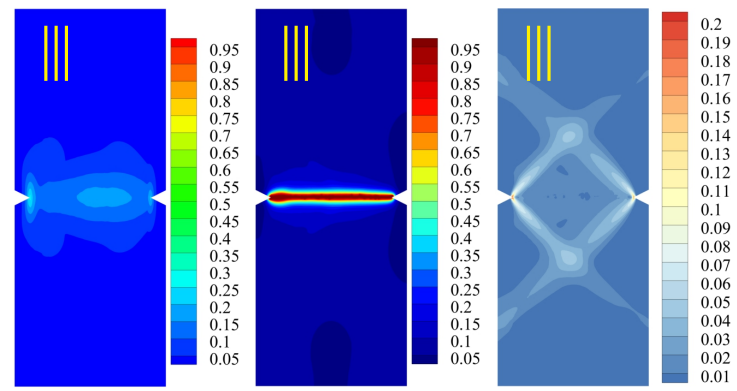

(d) $\theta=90^{\circ}$

Figure 15: Two-notch specimen. Final interfacial damage, bulk layer crack and equivalent plastic strain field for different layer orientations. 


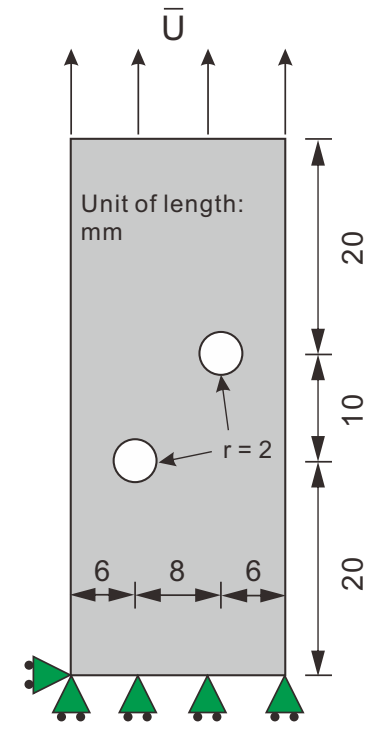

(a)

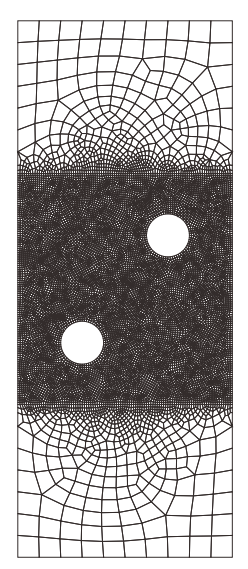

(b)

Figure 16: Two-hole specimen: (a) geometry and boundary conditions; (b) finite element model.

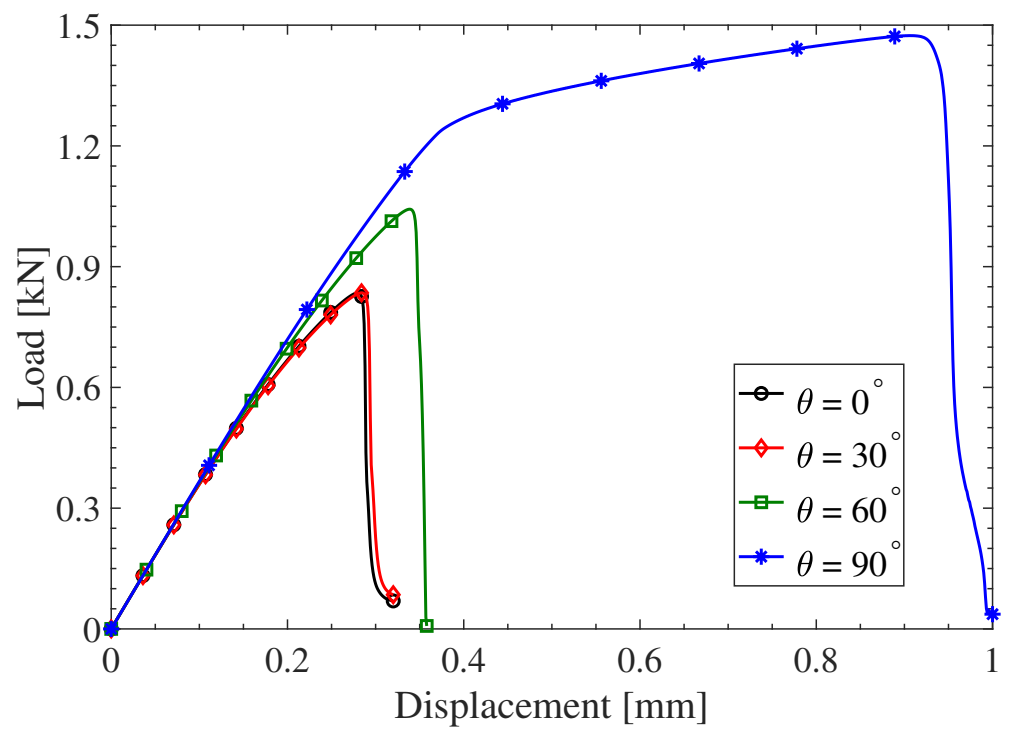

Figure 17: Two-hole specimen. Load-displacement curves for different layer orientations. 


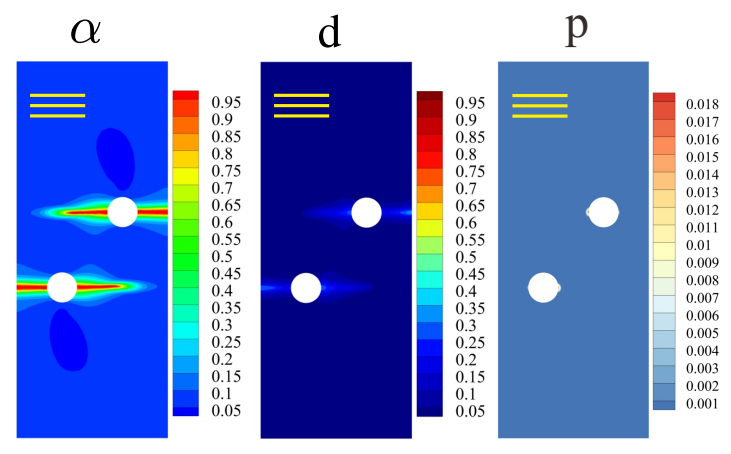

(a) $\theta=0^{\circ}$

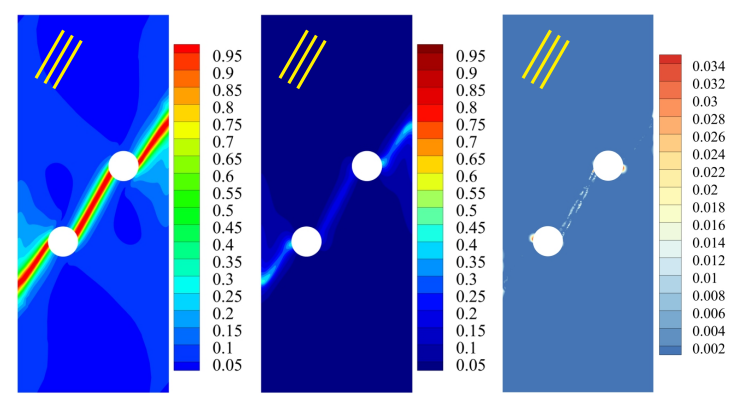

(c) $\theta=60^{\circ}$

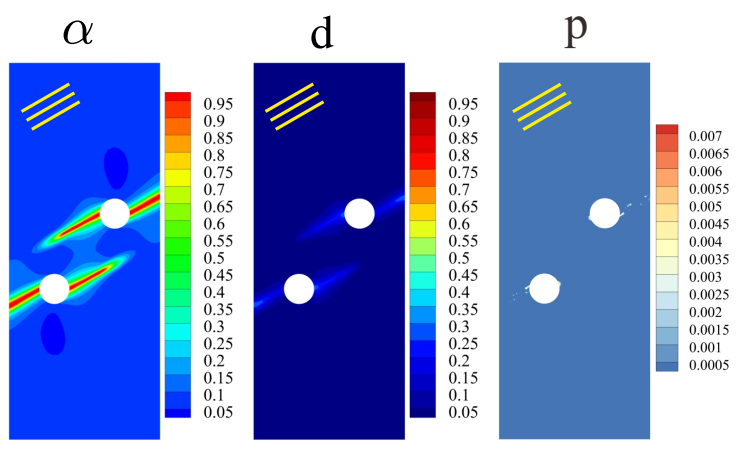

(b) $\theta=30^{\circ}$

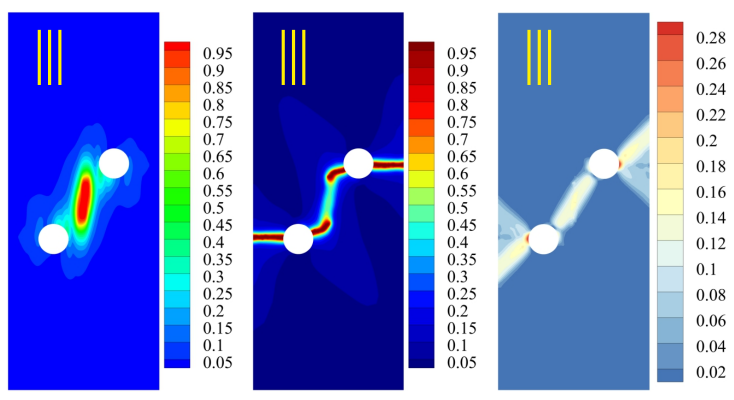

(d) $\theta=90^{\circ}$

Figure 18: Two-hole specimen. Final interfacial damage, bulk layer crack and equivalent plastic strain field for different layer orientations. 


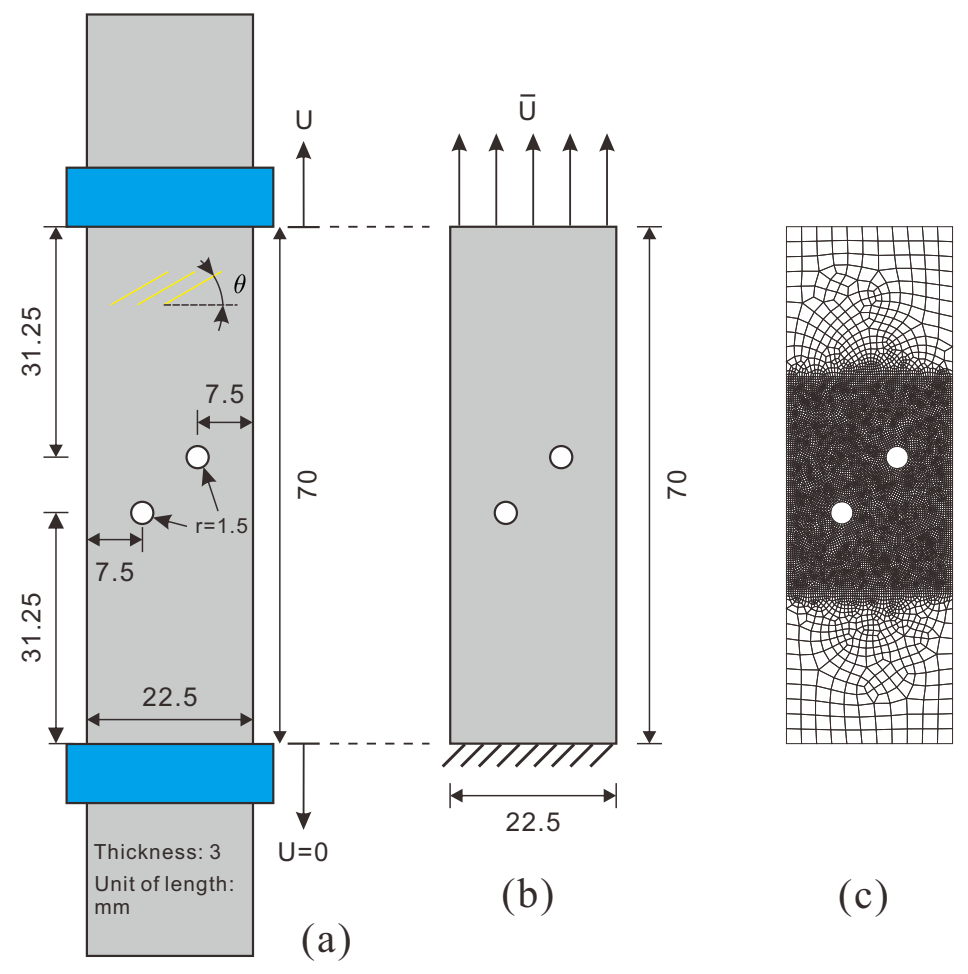

Figure 19: (a) Geometry and boundary conditions for experiments, (b) boundary conditions and (c) mesh model used in the numerical simulations.

\subsection{Experimental validation}

In this last example, the proposed anisotropic elastoplastic model is validated through experimental traction results on 3D printed samples.

The considered material consists of 3D printed Polyamide (Nylon) 12 material provided by Sinterit company and printed by SLS technique. The SLS 3D printing process consists in sintering the PA12 powder with the help of an infrared laser under elevated temperature. The high temperature helps the grains of the powder to consolidate before being bound with the laser beam. The powder is spread in thin even layers using a roller (see Fig. 1(a)) which also helps decreasing porosity between layers. The samples have been printed at MSME Lab on the LISA Sinterit printing machine using standard printing parameters provided by the manufacturer on the slicing software Sinterit Studio. These parameters include a layer thickness in the printing direction of $0.125 \mathrm{~mm}$. This machine uses an infrared laser diode to sinter the powder, the power of which is not accessible to the user and a temperature in the printing chamber of $170^{\circ} \mathrm{C}$. The orientation of the layers is defined by the position of the sample with respect to the plane where the laser sinters the powder. The samples are defined in Fig. 19, as well as the corresponding model and mesh used for the simulations. Three orientations of the layers with respect to the main loading have been considered, $\theta=0^{\circ}, \theta=45^{\circ}$ and $\theta=90^{\circ}$.

In the simulations, the loading is applied by displacement control through a series of load increments with a fixed step value $\Delta \bar{U}=5 \times 10^{-3} \mathrm{~mm}$. The spatial discretization of the model comprises 10,105 4-node quadrilateral elements, with refinement in the central region where the 


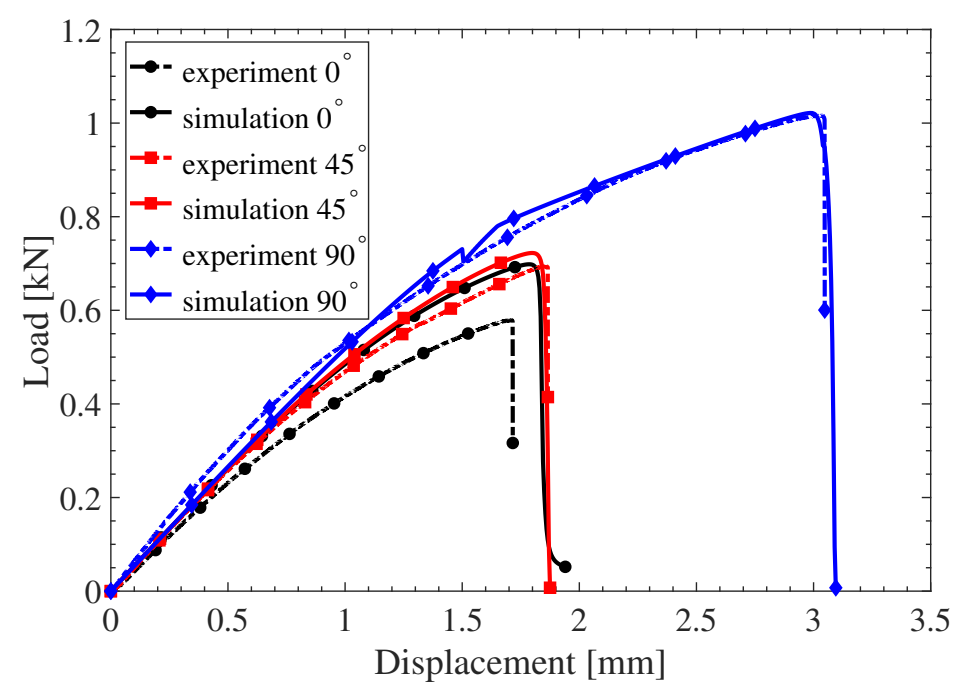

Figure 20: Comparison of the load-displacement curves obtained from experiments and numerical simulations.

crack is expected to propagate (see Fig. 19(c)). The following material parameters of the model have been identified using the experimental curves. We obtain: $E=0.57 \mathrm{GPa}, \nu=0.25, \sigma_{y}=0.01$ GPa and $H=0.28 \mathrm{GPa}$. The fracture toughness for bulk fracture and interfacial damage are $g_{c}^{d}=4.5 \times 10^{-3} \mathrm{kN} / \mathrm{mm}$ and $g_{c}^{\alpha}=1.7 \times 10^{-3} \mathrm{kN} / \mathrm{mm}$, respectively. The length scale parameters for bulk damage and interfacial damage are $\ell_{d}=\ell_{\alpha}=0.6 \mathrm{~mm}$. Here $\overline{\mathbf{C}}^{\alpha}$ is identified with the interphase RVE.

Fig. 20 shows the comparison of the load-displacement curves for experiments and numerical simulations. We can show that the present model is able to capture the experimentally observed transition from quasi-brittle behavior to elastoplastic-brittle behavior of the material and the right dependence to the orientation of the layers.

Finally, we present qualitative comparisons of crack patterns obtained from simulations and experiments in Fig. 21. In the experiments, only one crack is noticed, while two cracks are found in the simulations. However, for the case $\theta=45^{\circ}$, we note an orientation of the crack as in the simulation, even though the angle is not the same. Several reasons can cause these discrepancies: (a) there are many local defects in the experimental samples (pores, irregular boundaries, local distribution of fibers) which are not modeled in the simulations. In addition, the geometry of the two holes is only approximated in the experiments, as the boundaries are not circular but have stair-shape due to the $3 \mathrm{D}$ printing process. This can induce additional defects in the vicinity of the holes and trigger cracks preferentially near one single hole unlike in the simulations. Finally, in the case $\theta=90^{\circ}$, where the simulation predicts a fracture purely due to the damage of the polymer matrix, we note that the crack profile is not as straight as in the case $\theta=45^{\circ}$, where the damage was purely due to the damage of the interphase. We believe that to deliver a fair comparison regarding the crack patterns, a statistical study on more samples should be performed, but was unfortunately not possible in the context of this work and is reported to future studies.

In the present model, the elastic parameters $E$ and $\nu$ mainly control the slope of the loaddisplacement curve in the elastic stage; $\sigma_{y}$ is related to the critical point of plastic stage; $H$ affects the slope of the plastic hardening stage and $g_{c}^{\alpha}$ and $g_{c}^{d}$ control the initiation and propagation of 

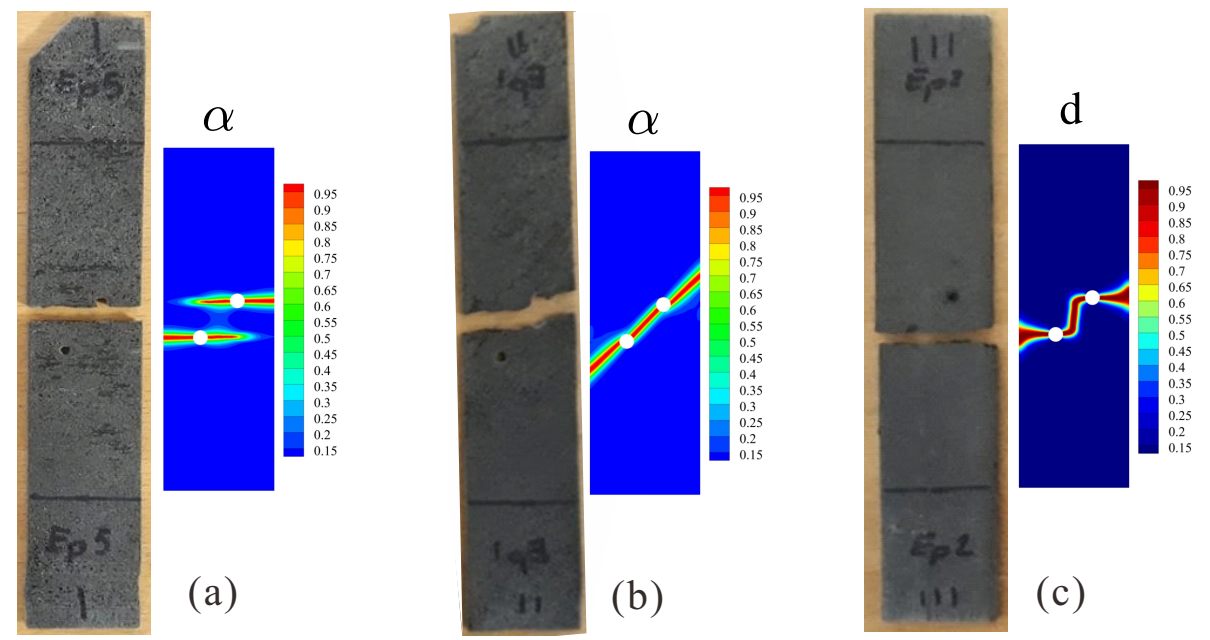

Figure 21: Comparison of the final cracks for experiments and numerical simulations: (a) $\theta=0^{\circ}$, (b) $\theta=45^{\circ}$ and (c) $\theta=90^{\circ}$.

interfacial damage and bulk layer damage, respectively. Thus, we used the following strategy for the identification of the material parameters: (i) we first identify the elastic parameters $E$ and $\nu$ using the elastic stage of the experimental load-displacement curves; (ii) we then identify the plastic parameters $\sigma_{y}$ and $H$ from the plastic hardening stage for $90^{\circ}$ load-displacement curve; (iii) we finally identify the damage parameters $g_{c}^{\alpha}$ and $g_{c}^{d}$ using the peak values of the experimental $45^{\circ}$ and $90^{\circ}$ load-displacement curves, respectively. We note that an inverse approach as proposed in [53] could also be employed to identify the material parameters in a more systematic manner, and could be investigated in a future work.

\section{Conclusion}

In this work, a phase field model for anisotropic, elastoplastic fracture model in layered structures obtained by 3D printing processes has been proposed. As compared to available anisotropic phase field models for fracture in the literature, three main contributions have been introduced. First, we have proposed an extension to elastoplasticity of anisotropic phase field model. Second, the model is able to describe a transition from quasi-brittle to elastoplastic fracture behaviors depending on the angle of layers in the microstructure with respect to the external loading. Such feature is of special interest to describe the anisotropic fracture behavior in layered 3D printed materials. The present model introduces two phase field variables, one bulk fracture damage variable and one micro interfacial damage variable, describing two different micro damage mechanisms. Finally, we have proposed an original methodology to identify the strain density function as a function of the micro interfacial damage variable using numerical homogenization on Representative Volume Elements.

The numerical investigations have shown that the present model is convergent with respect to mesh refinement, and allows to describe complex crack initiation and propagation in layered elastoplastic structures. An experimental comparison has been provided to validate the use of such model for $3 \mathrm{D}$ printed polymer materials. 
Possible extensions of this work include exploring more complex Representative Volume Elements configurations, as well as a fully nonlinear description of the strain density function as a function of the micro interfacial damage variable.

\section{Acknowledgements}

This work has benefited from the project "Multiscale Modeling and Experimental Investigation of damage in composite components obtained by additive manufacturing process" (MMELED), ANR-16-CE08-0044-04.

\section{References}

[1] L. S. Dimas, G. H. Bratzel, I. Eylon, M. J. Buehler, Tough composites inspired by mineralized natural materials: computation, 3D printing, and testing, Advanced Functional Materials 23 (36) (2013) 4629-4638.

[2] A. R. T. Perez, D. A. Roberson, R. B. Wicker, Fracture surface analysis of 3D-printed tensile specimens of novel abs-based materials, Journal of Failure Analysis and Prevention 14 (3) (2014) 343-353.

[3] N. Aliheidari, R. Tripuraneni, A. Ameli, S. Nadimpalli, Fracture resistance measurement of fused deposition modeling 3D printed polymers, Polymer Testing 60 (2017) 94-101.

[4] J. Russ, V. Slesarenko, S. Rudykh, H. Waisman, Rupture of 3D-printed hyperelastic composites: Experiments and phase field fracture modeling, Journal of the Mechanics and Physics of Solids 140 (2020) 103941.

[5] C. Richter, H. Lipson, Untethered hovering flapping flight of a 3D-printed mechanical insect, Artificial life 17 (2) (2011) 73-86.

[6] V. Mironov, T. Boland, T. Trusk, G. Forgacs, R. R. Markwald, Organ printing: computer-aided jet-based 3D tissue engineering, TRENDS in Biotechnology 21 (4) (2003) 157-161.

[7] H. Seitz, W. Rieder, S. Irsen, B. Leukers, C. Tille, Three-dimensional printing of porous ceramic scaffolds for bone tissue engineering, Journal of Biomedical Materials Research Part B: Applied Biomaterials: An Official Journal of The Society for Biomaterials, The Japanese Society for Biomaterials, and The Australian Society for Biomaterials and the Korean Society for Biomaterials 74 (2) (2005) 782-788.

[8] S. L. Rahim, S. Maidin, Feasibility study of additive manufacturing technology implementation in malaysian automotive industry using analytic hierarchy process, in: Advanced Materials Research, Vol. 903, Trans Tech Publ, 2014, pp. 450-454.

[9] J.-Y. Lee, W. S. Tan, J. An, C. K. Chua, C. Y. Tang, A. G. Fane, T. H. Chong, The potential to enhance membrane module design with 3D printing technology, Journal of Membrane Science 499 (2016) 480-490.

[10] S. H. Masood, Intelligent rapid prototyping with fused deposition modelling, Rapid Prototyping Journal (1996).

[11] L. Lü, J. Fuh, Y. Wong, Selective laser sintering, in: Laser-Induced Materials and Processes for Rapid Prototyping, Springer, 2001, pp. 89-142.

[12] R. Zou, Y. Xia, S. Liu, P. Hu, W. Hou, Q. Hu, C. Shan, Isotropic and anisotropic elasticity and yielding of 3D printed material, Composites Part B: Engineering 99 (2016) 506-513.

[13] A. Sakly, Fabrication additive de pièces à base d'alliages métalliques complexes, Ph.D. thesis, Université de Lorraine (2013).

[14] U. Cicekli, G. Z. Voyiadjis, R. K. A. Al-Rub, A plasticity and anisotropic damage model for plain concrete, International Journal of plasticity 23 (10-11) (2007) 1874-1900.

[15] L. Pelà, M. Cervera, P. Roca, Continuum damage model for orthotropic materials: Application to masonry, Computer Methods in Applied Mechanics and Engineering 200 (9-12) (2011) 917-930.

[16] M. Cai, P. Kaiser, Numerical simulation of the brazilian test and the tensile strength of anisotropic rocks and rocks with pre-existing cracks, International Journal of Rock Mechanics and Mining Sciences 41 (2004) 478-483.

[17] C. Ludwig, F. Rabold, M. Kuna, M. Schurig, H. Schlums, Simulation of anisotropic crack growth behavior of nickel base alloys under thermomechanical fatigue, Engineering Fracture Mechanics 224 (2020) 106800.

[18] E. M. Feerick, X. C. Liu, P. McGarry, Anisotropic mode-dependent damage of cortical bone using the Extended Finite Element Mmethod (XFEM), Journal of the mechanical behavior of biomedical materials 20 (2013) 77-89.

[19] D. Motamedi, S. Mohammadi, Dynamic crack propagation analysis of orthotropic media by the Extended Finite Element Method, International Journal of Fracture 161 (1) (2010) 21.

[20] G. Hattori, R. Rojas-Díaz, A. Sáez, N. Sukumar, F. García-Sánchez, New anisotropic crack-tip enrichment functions for the extended finite element method, Computational Mechanics 50 (5) (2012) 591-601. 
[21] W. Gao, X. Liu, S. Chen, T. Q. Bui, S. Yoshimura, A cohesive zone based de/fe coupling approach for interfacial debonding analysis of laminated glass, Theoretical and Applied Fracture Mechanics 108 (2020) 102668.

[22] S. Chen, H. Chen, N. Mitsume, N. Morita, T. Q. Bui, W. Gao, S. Yoshimura, A nodal-based Lagrange multiplier/cohesive zone approach for dynamic interfacial cracking analysis of thin-walled laminated composite structures, Composite Structures 256 (2021) 113112.

[23] I. Scheider, W. Brocks, Simulation of cup-cone fracture using the cohesive model, Engineering Fracture Mechanics 70 (14) (2003) 1943-1961.

[24] G. A. Francfort, J.-J. Marigo, Revisiting brittle fracture as an energy minimization problem, Journal of the Mechanics and Physics of Solids 46 (8) (1998) 1319-1342.

[25] B. Bourdin, G. A. Francfort, J.-J. Marigo, The variational approach to fracture, Journal of Elasticity 91 (1-3) (2008) 5-148.

[26] C. Miehe, M. Hofacker, F. Welschinger, A phase field model for rate-independent crack propagation: Robust algorithmic implementation based on operator splits, Computer Methods in Applied Mechanics and Engineering 199 (45-48) (2010) 2765-2778.

[27] C. Miehe, F. Welschinger, M. Hofacker, Thermodynamically consistent phase-field models of fracture: Variational principles and multi-field fe implementations, International Journal for Numerical Methods in Engineering 83 (10) (2010) 1273-1311.

[28] M. J. Borden, C. V. Verhoosel, M. A. Scott, T. J. Hughes, C. M. Landis, A phase-field description of dynamic brittle fracture, Computer Methods in Applied Mechanics and Engineering 217 (2012) 77-95.

[29] M. Ambati, T. Gerasimov, L. De Lorenzis, A review on phase-field models of brittle fracture and a new fast hybrid formulation, Computational Mechanics 55 (2) (2015) 383-405.

[30] T. T. Nguyen, J. Yvonnet, Q.-Z. Zhu, M. Bornert, C. Chateau, A phase field method to simulate crack nucleation and propagation in strongly heterogeneous materials from direct imaging of their microstructure, Engineering Fracture Mechanics 139 (2015) 18-39.

[31] E. Tanné, T. Li, B. Bourdin, J.-J. Marigo, C. Maurini, Crack nucleation in variational phase-field models of brittle fracture, Journal of the Mechanics and Physics of Solids 110 (2018) 80-99.

[32] J.-Y. Wu, A unified phase-field theory for the mechanics of damage and quasi-brittle failure, Journal of the Mechanics and Physics of Solids 103 (2017) 72-99.

[33] B. Bourdin, C. J. Larsen, C. L. Richardson, A time-discrete model for dynamic fracture based on crack regularization, International journal of fracture 168 (2) (2011) 133-143.

[34] G. Liu, Q. Li, M. A. Msekh, Z. Zuo, Abaqus implementation of monolithic and staggered schemes for quasi-static and dynamic fracture phase-field model, Computational Materials Science 121 (2016) 35-47.

[35] C. Miehe, L.-M. Schaenzel, H. Ulmer, Phase field modeling of fracture in multi-physics problems. part i. balance of crack surface and failure criteria for brittle crack propagation in thermo-elastic solids, Computer Methods in Applied Mechanics and Engineering 294 (2015) 449-485.

[36] J.-Y. Wu, T. K. Mandal, V. P. Nguyen, A phase-field regularized cohesive zone model for hydrogen assisted cracking, Computer Methods in Applied Mechanics and Engineering 358 (2020) 112614.

[37] Z. A. Wilson, C. M. Landis, Phase-field modeling of hydraulic fracture, Journal of the Mechanics and Physics of Solids 96 (2016) 264-290.

[38] C. Miehe, S. Mauthe, Phase field modeling of fracture in multi-physics problems. Part III. crack driving forces in hydro-poro-elasticity and hydraulic fracturing of fluid-saturated porous media, Computer Methods in Applied Mechanics and Engineering 304 (2016) 619-655.

[39] L. Xia, J. Yvonnet, S. Ghabezloo, Phase field modeling of hydraulic fracturing with interfacial damage in highly heterogeneous fluid-saturated porous media, Engineering Fracture Mechanics 186 (2017) 158-180.

[40] L. Xia, D. Da, J. Yvonnet, Topology optimization for maximizing the fracture resistance of quasi-brittle composites, Computer Methods in Applied Mechanics and Engineering 332 (2018) 234-254.

[41] J. B. Russ, H. Waisman, Topology optimization for brittle fracture resistance, Computer Methods in Applied Mechanics and Engineering 347 (2019) 238-263.

[42] D. Da, J. Yvonnet, L. Xia, G. Li, Topology optimization of particle-matrix composites for optimal fracture resistance taking into account interfacial damage, International Journal for Numerical Methods in Engineering 115 (5) (2018) 604-626.

[43] P. Li, Y. Wu, J. Yvonnet, A simp-phase field topology optimization framework to maximize quasi-brittle fracture resistance of 2d and 3d composites, Theoretical and Applied Fracture Mechanics 114 (2021) 102919.

[44] T.-T. Nguyen, J. Yvonnet, Q.-Z. Zhu, M. Bornert, C. Chateau, A phase-field method for computational modeling of interfacial damage interacting with crack propagation in realistic microstructures obtained by microtomography, Computer Methods in Applied Mechanics and Engineering 312 (2016) 567-595. 
[45] T.-T. Nguyen, J. Yvonnet, D. Waldmann, Q.-C. He, Phase field modeling of interfacial damage in heterogeneous media with stiff and soft interphases, Engineering Fracture Mechanics 218 (2019) 106574.

[46] P. Li, J. Yvonnet, C. Combescure, An extension of the phase field method to model interactions between interfacial damage and brittle fracture in elastoplastic composites, International Journal of Mechanical Sciences (2020) 105633.

[47] C. Wu, J. Fang, Z. Zhang, A. Entezari, G. Sun, M. V. Swain, Q. Li, Fracture modeling of brittle biomaterials by the phase-field method, Engineering Fracture Mechanics 224 (2020) 106752.

[48] M. Ambati, T. Gerasimov, L. De Lorenzis, Phase-field modeling of ductile fracture, Computational Mechanics 55 (5) (2015) 1017-1040.

[49] C. Miehe, M. Hofacker, L.-M. Schänzel, F. Aldakheel, Phase field modeling of fracture in multi-physics problems. Part II. Coupled brittle-to-ductile failure criteria and crack propagation in thermo-elastic-plastic solids, Computer Methods in Applied Mechanics and Engineering 294 (2015) 486-522.

[50] M. J. Borden, T. J. Hughes, C. M. Landis, A. Anvari, I. J. Lee, A phase-field formulation for fracture in ductile materials: Finite deformation balance law derivation, plastic degradation, and stress triaxiality effects, Computer Methods in Applied Mechanics and Engineering 312 (2016) 130-166.

[51] R. Alessi, J.-J. Marigo, S. Vidoli, Gradient damage models coupled with plasticity: variational formulation and main properties, Mechanics of Materials 80 (2015) 351-367.

[52] B. Yin, M. Kaliske, A ductile phase-field model based on degrading the fracture toughness: Theory and implementation at small strain, Computer Methods in Applied Mechanics and Engineering 366 (2020) 113068.

[53] T. T. Nguyen, J. Yvonnet, M. Bornert, C. Chateau, Initiation and propagation of complex 3D networks of cracks in heterogeneous quasi-brittle materials: Direct comparison between in situ testing-microct experiments and phase field simulations, Journal of the Mechanics and Physics of Solids 95 (2016) 320-350.

[54] Z.-J. Yang, B.-B. Li, J.-Y. Wu, X-ray computed tomography images based phase-field modeling of mesoscopic failure in concrete, Engineering Fracture Mechanics 208 (2019) 151-170.

[55] J.-Y. Wu, V. P. Nguyen, C. T. Nguyen, D. Sutula, S. Bordas, S. Sinaie, Phase field modeling of fracture, Advances in applied mechanics: multi-scale theory and computation 52 (2018).

[56] B. Li, C. Peco, D. Millán, I. Arias, M. Arroyo, Phase-field modeling and simulation of fracture in brittle materials with strongly anisotropic surface energy, International Journal for Numerical Methods in Engineering 102 (3-4) (2015) 711-727.

[57] S. Teichtmeister, D. Kienle, F. Aldakheel, M.-A. Keip, Phase field modeling of fracture in anisotropic brittle solids, International Journal of Non-Linear Mechanics 97 (2017) 1-21.

[58] B. Li, C. Maurini, Crack kinking in a variational phase-field model of brittle fracture with strongly anisotropic surface energy, Journal of the Mechanics and Physics of Solids 125 (2019) 502-522.

[59] N. Noii, F. Aldakheel, T. Wick, P. Wriggers, An adaptive global-local approach for phase-field modeling of anisotropic brittle fracture, Computer Methods in Applied Mechanics and Engineering 361 (2020) 112744.

[60] A. Quintanas-Corominas, J. Reinoso, E. Casoni, A. Turon, J. Mayugo, A phase field approach to simulate intralaminar and translaminar fracture in long fiber composite materials, Composite Structures 220 (2019) 899-911.

[61] P. Zhang, X. Hu, T. Q. Bui, W. Yao, Phase field modeling of fracture in fiber reinforced composite laminate, International Journal of Mechanical Sciences 161 (2019) 105008.

[62] J. Clayton, J. Knap, Phase field modeling of directional fracture in anisotropic polycrystals, Computational Materials Science 98 (2015) 158-169.

[63] T.-T. Nguyen, J. Réthoré, J. Yvonnet, M.-C. Baietto, Multi-phase-field modeling of anisotropic crack propagation for polycrystalline materials, Computational Mechanics 60 (2) (2017) 289-314.

[64] P. Shanthraj, B. Svendsen, L. Sharma, F. Roters, D. Raabe, Elasto-viscoplastic phase field modelling of anisotropic cleavage fracture, Journal of the Mechanics and Physics of Solids 99 (2017) 19-34.

[65] M. Paggi, M. Corrado, J. Reinoso, Fracture of solar-grade anisotropic polycrystalline silicon: A combined phase field-cohesive zone model approach, Computer Methods in Applied Mechanics and Engineering 330 (2018) $123-148$.

[66] X. Zhang, S. W. Sloan, C. Vignes, D. Sheng, A modification of the phase-field model for mixed mode crack propagation in rock-like materials, Computer Methods in Applied Mechanics and Engineering 322 (2017) 123136.

[67] E. C. Bryant, W. Sun, A mixed-mode phase field fracture model in anisotropic rocks with consistent kinematics, Computer Methods in Applied Mechanics and Engineering 342 (2018) 561-584.

[68] R. Alessi, F. Freddi, Phase-field modelling of failure in hybrid laminates, Composite Structures 181 (2017) 9-25.

[69] R. Alessi, F. Freddi, Failure and complex crack patterns in hybrid laminates: A phase-field approach, Composites 
Part B: Engineering 179 (2019) 107256.

[70] Q.-C. H. Q. Shao, Closed-form coordinate-free decompositions of the two-dimensional strain and stress for modeling tension-compression dissymmetry, Journal of Applied Mechanics 86 (3) (2019) 031007.

[71] T.-T. Nguyen, J. Yvonnet, D. Waldmann, Q.-C. He, Implementation of a new strain split to model unilateral contact within the phase field method, International Journal for Numerical Methods in Engineering 121 (21) (2020) 4717-4733.

[72] J. Bleyer, R. Alessi, Phase-field modeling of anisotropic brittle fracture including several damage mechanisms, Computer Methods in Applied Mechanics and Engineering 336 (2018) 213-236.

[73] B. Yin, M. Kaliske, An anisotropic phase-field model based on the equivalent crack surface energy density at finite strain, Computer Methods in Applied Mechanics and Engineering 369 (2020) 113202.

[74] T. Nguyen, J. Yvonnet, M. Bornert, C. Chateau, K. Sab, R. Romani, R. L. Roy, On the choice of parameters in the phase field method for simulating crack initiation with experimental validation, Int. J. Fract. 197 (2) (2016) $213-226$.

[75] T. K. Mandal, A. Gupta, V. P. Nguyen, R. Chowdhury, A. de Vaucorbeil, A length scale insensitive phase field model for brittle fracture of hyperelastic solids, Engineering Fracture Mechanics 236 (2020) 107196.

[76] T. K. Mandal, V. P. Nguyen, J.-Y. Wu, A length scale insensitive anisotropic phase field fracture model for hyperelastic composites, International Journal of Mechanical Sciences 188 (2020) 105941.

[77] P. Rodriguez, J. Ulloa, C. Samaniego, E. Samaniego, A variational approach to the phase field modeling of brittle and ductile fracture, International Journal of Mechanical Sciences 144 (2018) 502-517.

[78] A. Mielke, Evolution of rate-independent systems, Evolutionary equations 2 (2005) 461-559.

[79] A. Mielke, A mathematical framework for generalized standard materials in the rate-independent case, in: Multifield Problems in Solid and Fluid Mechanics, Springer, 2006, pp. 399-428.

[80] K. Pham, J.-J. Marigo, C. Maurini, The issues of the uniqueness and the stability of the homogeneous response in uniaxial tests with gradient damage models, Journal of the Mechanics and Physics of Solids 59 (6) (2011) $1163-1190$.

[81] J. Simo, T. Hughes, Computational Inelasticity, Springer-Verlag, 1998.

[82] F. P. Duda, A. Ciarbonetti, P. J. Sánchez, A. E. Huespe, A phase-field/gradient damage model for brittle fracture in elastic-plastic solids, International Journal of Plasticity 65 (2015) 269-296.

[83] C. Miehe, F. Aldakheel, A. Raina, Phase field modeling of ductile fracture at finite strains: A variational gradient-extended plasticity-damage theory, International Journal of Plasticity 84 (2016) 1-32.

[84] J. Yvonnet, Computational Homogenization of Heterogeneous Materials with Finite Elements, Springer, 2019.

[85] T. Zohdi, P. Wriggers, An introduction to computational micromechanics, Springer Science \& Business Media, 2008.

[86] E. A. de Souza Neto, D. Peric, D. R. Owen, Computational methods for plasticity: theory and applications, John Wiley \& Sons, 2011. 